\title{
JORDAN DECOMPOSITIONS OF COCENTERS OF REDUCTIVE $p$-ADIC GROUPS
}

\author{
XUHUA HE AND JU-LEE KIM
}

\begin{abstract}
Cocenters of Hecke algebras $\mathcal{H}$ play an important role in studying $\bmod \ell$ or $\mathbb{C}$ harmonic analysis on connected $p$-adic reductive groups. On the other hand, the depth $r$ Hecke algebra $\mathcal{H}_{r^{+}}$is well suited to study depth $r$ smooth representations. In this paper, we study depth $r$ rigid cocenters $\overline{\mathcal{H}}_{r+}^{\text {rig }}$ of a connected reductive $p$-adic group over rings of characteristic zero or $\ell \neq p$. More precisely, under some mild hypotheses, we establish a Jordan decomposition of the depth $r$ rigid cocenter, hence find an explicit basis of $\overline{\mathcal{H}}_{r+}^{\text {rig }}$.
\end{abstract}

\section{INTRODUCTION}

0.1 . Let $G$ be a connected reductive $p$-adic group. Let $R$ be an algebraically closed field of characteristic not equal to $p$. Let $\mathcal{H}_{R}$ be the Hecke algebra of locally constant, compactly supported $R$-valued functions on $G$. The trace map

$$
\operatorname{Tr}_{R}: \overline{\mathcal{H}}_{R} \rightarrow \mathfrak{R}_{R}(G)^{*}
$$

relates the cocenter $\overline{\mathcal{H}}_{R}=\mathcal{H}_{R} /\left[\mathcal{H}_{R}, \mathcal{H}_{R}\right]$ and the Grothendieck group $\mathfrak{R}_{R}(G)$ of smooth admissible representations of $G$ over $R$.

In most cases, the cocenter is expected to be "dual" to the representations. For $R=\mathbb{C}$, Bernstein, Deligne, and Kazhdan in [5] and [16] proved that the trace map $\operatorname{Tr}_{\mathbb{C}}: \overline{\mathcal{H}}_{\mathbb{C}} \cong \mathfrak{R}_{\mathbb{C}}(G)_{\text {good }}^{*}$ is a bijection between the cocenter and the "good linear forms" on $\mathfrak{R}_{\mathbb{C}}(G)$. For modular representations over $R$, the surjection $\operatorname{Tr}_{R}: \overline{\mathcal{H}}_{R} \rightarrow$ $\mathfrak{R}_{R}(G)_{\text {good }}^{*}$ is established in [6] under the assumption that the cardinality of the relative Weyl group of $G$ is invertible in $R$. It is conjectured that the injection holds if the pro- $p$ order of any open compact subgroup of $G$ is invertible in $R$.

This motivates our study of the structure of the cocenter of the Hecke algebra. To be precise, we mainly consider the integral form $\mathcal{H}=\mathcal{H}(G)$, i.e., $\mathcal{H}_{R}(G)$ with $R=\mathbb{Z}\left[\frac{1}{p}\right]$. This will allow us to apply the results on $\overline{\mathcal{H}}$ to both the ordinary and the modular representations of $G$.

0.2. In [12, Theorem B] and [13, Theorems $\mathrm{C}$ and 6.5], the first-named author showed that

$$
\overline{\mathcal{H}} \cong \bigoplus_{M} \overline{\mathcal{H}}(M)^{\text {rig, }+}
$$

where $M$ runs over all the standard Levi subgroups of $G$ and $\overline{\mathcal{H}}(M)^{\text {rig, }+}$ is the +rigid part of the cocenter of the Hecke algebra $\mathcal{H}(M)$, i.e., the $\mathbb{Z}\left[\frac{1}{p}\right]$-submodule

Received by the editors October 17, 2017, and, in revised form, October 30, 2018.

2010 Mathematics Subject Classification. Primary 22E50; Secondary 11F70.

The first author was partially supported by NSF DMS-1463852 and DMS-1128155 (from IAS). 
of $\overline{\mathcal{H}}(M)$ consisting of elements represented by the functions supported in the compact-modulo-center elements of $M$ whose Newton points are dominant (in $G$ ) and with centralizer equal to $M$. In other words, the rigid cocenters of the Hecke algebras of various standard Levi subgroups form the "building block" of the whole cocenter $\overline{\mathcal{H}}$. We refer to 12,13 for the details.

Note that if $M=G$, then we write $\overline{\mathcal{H}}^{\text {rig }}$ for $\overline{\mathcal{H}}(G)^{\text {rig, }+}$, the $\mathbb{Z}\left[\frac{1}{p}\right]$-submodule of $\overline{\mathcal{H}}$ represented by functions supported in the subset $G^{\text {rig }}$ of compact-modulo-center elements of $G$. We call $\overline{\mathcal{H}}^{\text {rig }}$ the rigid cocenter. In this paper, we focus on the depth $r$ rigid cocenter $\overline{\mathcal{H}}_{r^{+}}^{\text {rig }}$ for any real number $r>0$, defined as follows.

For any element $x$ in the reduced Bruhat-Tits building $\mathcal{B}(G)$ of $G$, Moy and Prasad 21] associated a subgroup $G_{x, r^{+}}$of $G$. Let $\mathcal{H}_{r^{+}}=\sum_{x \in \mathcal{B}(G)} C_{c}\left(G / G_{x, r^{+}}\right)$ and $\overline{\mathcal{H}}_{r+}$ be its image in $\overline{\mathcal{H}}$, the depth $r$ cocenter. The depth $r$ rigid cocenter $\overline{\mathcal{H}}_{r^{+}}^{\text {rig }}=\overline{\mathcal{H}}^{\text {rig }} \cap \overline{\mathcal{H}}_{r^{+}}$. According to Howe's conjecture (now proved in [4,7,12]), this is a finitely generated $\mathbb{Z}\left[\frac{1}{p}\right]$-module. Moreover, we have that $\overline{\mathcal{H}}^{\text {rig }}=\lim _{r} \overline{\mathcal{H}}_{r^{+}}^{\text {rig }}$.

The main purpose of this paper is to establish the "Jordan decomposition" of $\overline{\mathcal{H}}_{r^{+}}^{\text {rig }}$. Note that the "Jordan decomposition" in the paper is compatible with taking the "+" part and thus apply to $\overline{\mathcal{H}}(M)^{\text {rig, }+}$ as well.

0.3. Before stating the main result, we make a short digression and discuss a "toy model", the cocenter of the group algebra $\mathbb{Z}[H]$ of a finite reductive group $H$.

For any element $g \in H$, we have the Jordan decomposition $g=g_{s} g_{u}$, where $g_{s}$ is the semisimple part of $g$ and $g_{u}$ is the unipotent part of $g$. Then we have the Jordan decomposition of the group algebra $\mathbb{Z}[H] \cong \bigoplus_{s \in H^{s s}} \mathbb{Z}\left[C_{H}(s)^{\text {unip }}\right]$, where $H^{s s}$ is the set of semisimple elements of $H, C_{H}(s)$ is the centralizer of $s$, and $C_{H}(s)^{\text {unip }}$ is the set of unipotent elements in $C_{H}(s)$. Based on the Jordan decomposition on the group algebra $\mathbb{Z}[H]$, one deduces the Jordan decomposition of the cocenter

$$
\overline{\mathbb{Z}[H]}:=\mathbb{Z}[H] /[\mathbb{Z}[H], \mathbb{Z}[H]] \cong \bigoplus_{[s] \in C l^{s s}(H)} \overline{\mathbb{Z}\left[C_{H}(s)^{\text {unip }}\right]},
$$

where $C l^{s s}(H)$ is the set of semisimple conjugacy classes of $H$ and $\overline{\mathbb{Z}\left[C_{H}(s)^{\text {unip }}\right]}$ is the image of $\mathbb{Z}\left[C_{H}(s)^{\text {unip }}\right]$ in $\overline{\mathbb{Z}\left[C_{H}(s)\right]}$, which is a free $\mathbb{Z}$-module with basis indexed by the unipotent conjugacy classes of $C_{H}(s)$.

0.4. Now we come back to connected reductive $p$-adic groups. As any element in the Hecke algebra $\mathcal{H}$ is a locally constant function, there seems no analogous Jordan decomposition on $\mathcal{H}$. However, under the hypotheses in $₫ 2.2$, we have the analogous part of semisimple conjugacy classes and unipotent conjugacy classes in the context of the cocenter of Hecke algebras.

By the work of Adler and Spice [3], we may write a semisimple compact-modulocenter element $\gamma$ as a "good product". Since we are working with the cocenter $\overline{\mathcal{H}}_{r^{+}}^{\text {rig }}$ of depth $r$, we use the truncated part $\gamma_{\leq r}$ of $\gamma$. The equivalence classes $\mathcal{S}_{r}$ of semisimple compact-modulo-center elements of $G$, roughly speaking, are generated by the conjugation action and the truncated operation (see $\$ 2$ for the precise definition). The set $\mathcal{S}_{r}$ is the analogue of semisimple conjugacy classes and serves as the index set of the desired Jordan decomposition on $\overline{\mathcal{H}}_{r^{+}}^{\text {rig }}$.

For any $[\gamma] \in \mathcal{S}_{r}$, we pick up the truncation $\gamma_{\leq r}$ (see Definition 2.4.4 for details) of a representative $\gamma \in[\gamma]$ and denote by $C_{G}\left(\gamma_{\leq r}\right)$ the centralizer of $\gamma_{\leq r}$. The 
isomorphism class of $C_{G}\left(\gamma_{\leq r}\right)$ is independent of the choice of $\gamma$ and its truncation $\gamma_{\leq r}$.

Now we come to the unipotent part. Let $\overline{\mathcal{H}}_{r^{+}}^{G, b}$ be the $\mathbb{Z}\left[\frac{1}{p}\right]$-submodule of $\overline{\mathcal{H}}_{r^{+}}^{\text {rig }}$, represented by functions in $\sum_{x \in \mathcal{B}(G)} C_{c}\left(G_{x, r} / G_{x, r^{+}}\right)$with support in

$$
G_{r^{+}}:=\bigcup_{x \in \mathcal{B}(G)} G_{x, r^{+}} .
$$

Based on the work of DeBacker in [8] and [9], $\overline{\mathcal{H}}_{r^{+}}^{G, b}$ is a free module with basis indexed by the unipotent conjugacy classes of $G$. This is the analogy of the set of unipotent conjugacy classes, or in other words, the analogy of $\overline{\mathbb{Z}\left[H^{\text {unip }}\right]}$ in the cocenter of the group algebra $\mathbb{Z}[H]$. Now we state the main results of this paper.

Theorem A (Theorems 3.1.2 and 4.2.1). Fix $r \in \mathbb{R}_{>0}$. Suppose the hypotheses in 2.2 hold. Then

$$
\overline{\mathcal{H}}_{r^{+}}^{\mathrm{rig}} \cong \bigoplus_{[\gamma] \in \mathcal{S}_{r}} \overline{\mathcal{H}}_{r^{+}}^{C_{G}\left(\gamma_{\leq r}\right), b} .
$$

Moreover, $\overline{\mathcal{H}}_{r+}^{\text {rig }}$ is a free $\mathbb{Z}\left[\frac{1}{p}\right]$-module.

Theorem B (Theorem 4.3.1). Let $J\left(G^{\mathrm{rig}}\right)$ denote the space of $\mathbb{C}$-valued invariant distributions of $G$ with support on $G^{\mathrm{rig}}$. Suppose the hypotheses in $\$ 2.2$ hold. Then the restriction $\left.J\left(G^{\mathrm{rig}}\right)\right|_{\mathcal{H}_{r+, c}}$ has a basis given by the restriction of orbital integrals $O_{\gamma_{\leq r} u}$ to $\mathcal{H}_{r^{+}, \mathbb{C}}$, where $[\gamma] \in \mathcal{S}_{r}$, and $u$ runs over the representatives of the unipotent conjugacy classes of $C_{G}\left(\gamma_{\leq r}\right)$.

0.5. In $\S 1$, we review some background material on Moy-Prasad filtration subgroups and the cocenter $\mathcal{H}$ of $G$. Toward the decomposition of $\overline{\mathcal{H}}_{r^{+}}^{\text {rig }}$ in Theorem $\mathrm{A}$, in $\S 2$, we first decompose $G^{\text {rig }}$ into a disjoint union of $G$-domains $X_{[\gamma]}:={ }^{G}\left(\gamma C_{G}\left(\gamma_{\leq r}\right)_{r^{+}}\right)$parameterized by $[\gamma] \in \mathcal{S}_{r}$. We use good products of semisimple elements $(\underline{3})$ to prove that $G^{\text {rig }}=\bigcup_{[\gamma] \in \mathcal{S}_{r}} X_{[\gamma]}$. The Lie algebra version of such decompositions can be found in [17, §7]. Then, it is easy to see that there is a corresponding decomposition of $\mathcal{H}^{\text {rig }}$ according to this decomposition: $\mathcal{H}^{\text {rig }}=\bigoplus_{[\gamma]} \mathcal{H}\left(X_{[\gamma]}\right)$ (Lemma 1.2.3) where $\mathcal{H}\left(X_{[\gamma]}\right)$ is the submodule consisting of $f \in \mathcal{H}_{r^{+}}$with $\operatorname{Supp}(f) \subset X_{[\gamma]}$. However, since each domain $X_{[\gamma]}$ is not necessarily $G_{x, r^{+}}$bi-invariant, $\bigoplus_{[\gamma] \in \mathcal{S}_{r}} \mathcal{H}_{r^{+}}\left(X_{[\gamma]}\right)$ is in fact a proper submodule of $\mathcal{H}_{r^{+}}^{\text {rig }}$ (see 3.1 ).

Now, Theorem A asserts that the desired decomposition holds at the level of cocenters. In 83 , we prove Theorem $\mathrm{A}$ via the following strategy: we first represent elements in $\overline{\mathcal{H}}_{r^{+}}^{\text {rig }}$ by elements in $\mathcal{H}_{0, r^{+}}:=\sum_{x \in \mathcal{B}(G)} C_{c}\left(Z(G) \operatorname{Stab}_{G}(x) / G_{x, r^{+}}\right)$in the cocenter, and then represent elements in $\overline{\mathcal{H}}_{0, r}+$ by elements in $\bigoplus_{[\gamma]} \overline{\mathcal{H}}\left(X_{[\gamma]}\right)$. In these steps, we use the descent arguments developed by Howe, Harish-Chandra, Waldspurger, and most recently by DeBacker. In particular, DeBacker's arguments in [9] are aptly adaptable in our situations in view of recent developments in harmonic analysis on $p$-adic groups. As a result, most of our hypotheses are inherited from [3] and [9]. Lastly, we prove $\overline{\mathcal{H}}_{r^{+}}\left(X_{[\gamma]}\right) \simeq \overline{\mathcal{H}}_{r^{+}}^{C_{G}\left(\gamma_{\leq r}\right), b}$ using inductive descents (see Proposition 3.4.1). A Lie algebra version of inductive descents can be found in [19, $\S 6]$.

In 44 , we prove Theorem $\mathrm{B}$. We combine inductive descents and the parameterization of unipotent conjugacy classes in [9. However, since the centralizer of a 
semisimple element is not necessarily connected in this paper, one needs to adapt DeBacker's parameterization for our case.

In §5, we present examples to illustrate the duality between cocenters and representations.

Notation and conventions. Let $F$ be a non-archimedean local field with finite residue field $\mathbb{F}_{p^{n}}$. Let $\mathbb{G}$ be a connected reductive group defined over $F$. For any finite extension $E$ of $F$, let $\mathbb{G}(E)$ be the group of $E$-rational points of $\mathbb{G}$. We will simply write $G$ for $\mathbb{G}(F)$. Denote the Lie algebras of $\mathbb{G}$ and $\mathbb{G}(E)$ by $\mathfrak{g}$ and $\mathfrak{g}(E)$, respectively.

In general, we use bold characters $\mathbb{H}, \mathbb{M}$, and $\mathbb{N}$, etc., to denote algebraic groups. If they are defined over $F$, we will use corresponding Roman characters $H, M$, and $N$ to denote the groups of $F$-points, and $\mathfrak{h}, \mathfrak{m}$, and $\mathfrak{n}$ to denote the Lie algebras of $H, M$, and $N$. Let $\overline{\mathbb{G}}:=\mathbb{G} / Z(\mathbb{G})$ where $Z(\mathbb{G})$ is the center of $\mathbb{G}$. We also denote the center of $G$ as $Z(G)$.

We denote by $G^{s s}$ the set of semisimple elements in $G$, by $\mathcal{U}$ the set of unipotent elements in $G$, and by $G^{\text {rig }}$ the set of compact-modulo-center elements in $G$.

We let $\mu_{G}$ denote a fixed Haar measure on $G$.

For $g \in G,{ }^{g} X$ denotes $g X g^{-1}$ and for $S, H \subset G,{ }^{H} S:=\left\{{ }^{g} X \mid X \in S, g \in H\right\}$.

We set $\tilde{\mathbb{R}}=\mathbb{R} \sqcup\left\{r^{+} ; r \in \mathbb{R}\right\} \sqcup\{\infty\}$ and define the partial order on $\tilde{\mathbb{R}}$ as follows: for $r, s \in \mathbb{R}, r<s^{+}$if $r \leq s, r^{+}<s^{+}$and $r^{+}<s$ if $r<s$, and $r, r^{+}<\infty$ for any $r \in \mathbb{R}$.

We denote by $\mathcal{H}$, the Hecke algebra of locally constant, compactly supported $\mathbb{Z}\left[\frac{1}{p}\right]$-valued functions on $G$. The cocenter $\overline{\mathcal{H}}=\mathcal{H} /[\mathcal{H}, \mathcal{H}]$. Let $\mathcal{H}^{\text {rig }}$ be the $\mathbb{Z}\left[\frac{1}{p}\right]$ submodule of $\mathcal{H}$ consisting of functions supported in $G^{\text {rig }}$. The rigid cocenter $\overline{\mathcal{H}}^{\text {rig }}$ is the image of $\mathcal{H}^{\text {rig }}$ in $\overline{\mathcal{H}}$.

\section{Preliminaries}

\subsection{Moy-Prasad filtrations.}

1.1.1. Apartments and buildings. For a finite extension $E$ of $F$, let $\mathcal{B}(\mathbb{G}, E)$ denote the extended Bruhat-Tits building of $\mathbb{G}$ over $E$. Recall that $\mathcal{B}(\mathbb{G}, E) \simeq$ $\mathcal{B}(\overline{\mathbb{G}}, E) \times\left(\mathrm{X}_{*}(Z(\mathbb{G}), E) \otimes \mathbb{R}\right)$, and $\mathrm{X}_{*}(Z(\mathbb{G}), E)$ is the abelian group of $E$-rational cocharacters of the center $Z(\mathbb{G})$ of $\mathbb{G}$.

If $\mathbb{T}$ is a maximal $F$-torus in $\mathbb{G}$ which splits over $E$, let $\mathcal{A}(\mathbb{T}, E)$ be the corresponding apartment over $E$. It is known that for any tamely ramified finite Galois extension $E^{\prime}$ of $E, \mathcal{B}(\mathbb{G}, E)$ can be embedded into $\mathcal{B}\left(\mathbb{G}, E^{\prime}\right)$ and its image is equal to the set of the Galois fixed points in $\mathcal{B}\left(\mathbb{G}, E^{\prime}\right)$ (see [25, (5.11)] or [23]).

For a maximal $F$-torus $\mathbb{T}$ in $\mathbb{G}$ which splits over a tamely ramified finite Galois extension $E$ of $F$, we write $\mathcal{A}(\mathbb{T}, F)$ for $\mathcal{A}(\mathbb{T}, E) \cap \mathcal{B}(\mathbb{G}, F)$. This is well defined independent of the choice of $E$. Moreover, $\mathcal{A}(\mathbb{T}, F)$ is the set of Galois fixed points in $\mathcal{A}(\mathbb{T}, E)$. For simplicity, we write $\mathcal{B}(G)=\mathcal{B}(\mathbb{G}, F), \mathcal{A}(T)=\mathcal{A}(\mathbb{T}, F)$, etc.

1.1.2. Moy-Prasad filtrations. Regarding $\mathbb{G}$ as a group defined over $E$, Moy and Prasad associate $\mathfrak{g}(E)_{x, r}$ and $\mathbb{G}(E)_{x,|r|}$ (resp., $\mathfrak{g}(E)_{x, r^{+}}$and $\mathbb{G}(E)_{x,|r|^{+}}$) to $(x, r) \in \mathcal{B}(\mathbb{G}, E) \times \mathbb{R}$ with respect to the valuation normalized as follows $(22)$ : let $E^{u}$ be the maximal unramified extension of $E$, and let $L$ be the minimal extension 
of $E^{u}$ over which $\mathbb{G}$ splits. Then the valuation used by Moy and Prasad maps $L^{\times}$ onto $\mathbb{Z}$.

In this paper, we let $\nu=\nu_{F}$ be the valuation on $F$ such that $\nu\left(F^{\times}\right)=\mathbb{Z}, \nu_{E}$ extends $\nu$. Let $\bar{F}$ be an algebraic closure of $F$. For an extension field $E$ of $F$, let $\nu_{E}$ be the valuation on $E$ extending $\nu$. We will just write $\nu$ for $\nu_{E}$. Then, with respect to our normalized valuation $\nu$, we can define filtrations in $\mathfrak{g}(E)$ and $\mathbb{G}(E)$. Then our $\mathfrak{g}(E)_{x, r}$ and $\mathbb{G}(E)_{x, r}$ correspond to $\mathfrak{g}(E)_{x, \text { elr }}$ and $\mathbb{G}(E)_{x, \text { elr }}$ of Moy and Prasad, where $e=e(E / F)$ is the ramification index of $E$ over $F$ and $l=\left[L: E^{u}\right]$.

This normalization is chosen to have the following property [1, (1.4.1)]:

(1) For a tamely ramified Galois extension $E^{\prime}$ of $E$ and $x \in \mathcal{B}(\mathbb{G}, E) \subset \mathcal{B}\left(\mathbb{G}, E^{\prime}\right)$, for $r \in \widetilde{\mathbb{R}}$, we have

$$
\mathfrak{g}(E)_{x, r}=\mathfrak{g}\left(E^{\prime}\right)_{x, r} \cap \mathfrak{g}(E) .
$$

If $r>0$,

$$
\mathbb{G}(E)_{x, r}=\mathbb{G}\left(E^{\prime}\right)_{x, r} \cap \mathbb{G}(E) .
$$

(2) For $r \in \frac{1}{e} \mathbb{Z}_{\geq 0}$, two points $x$ and $y$ in $\mathcal{B}(\mathbb{G}, E)$ lie in the same facet if and only if

$$
\mathbb{G}(E)_{x, r}=\mathbb{G}(E)_{y, r} \quad \text { and } \quad \mathbb{G}(E)_{x, r^{+}}=\mathbb{G}(E)_{y, r^{+}} .
$$

1.1.3. For simplicity, we put $G_{x, r}:=\mathbb{G}(F)_{x, r}$, etc. We will also use the following notation. For $r \in \mathbb{R}_{\geq 0}$, let

$$
G_{r}=\bigcup_{x \in \mathcal{B}(G)} G_{x, r}, \quad G_{r^{+}}=\bigcup_{s>r} G_{s} .
$$

Let $\Phi(\mathbb{T}, \mathbb{G}, E)$ be the set of $E$-roots of $\mathbb{T}$ in $\mathbb{G}$, and let $\Psi(\mathbb{T}, \mathbb{G}, E)$ be the corresponding set of affine roots in $\mathbb{G}$. If $\psi \in \Psi(\mathbb{T}, \mathbb{G}, E)$, let $\dot{\psi} \in \Phi(\mathbb{T}, \mathbb{G}, E)$ be the gradient of $\psi$, and let $\mathbb{U}(E)_{\dot{\psi}} \subset \mathbb{G}(E)$ be the root group corresponding to $\dot{\psi}$. We denote the root subgroup in $\mathbb{U}(E)_{\dot{\psi}}$ corresponding to $\psi$ by $\mathbb{U}(E)_{\psi}$.

Let $\mathrm{X}_{*}(\mathbb{T}, E)$ be the set of cocharacters of $\mathbb{T}$, and let $\mathrm{X}^{*}(\mathbb{T}, E)$ be the set of characters of $\mathbb{T}$. Let $T_{0}$ be the maximal compact subgroup of $T$. For $r \geq 0$, set

$$
\begin{aligned}
& T_{r}:=\left\{t \in T_{0} \mid \nu(\chi(t)-1) \geq r \text { for all } \chi \in \mathrm{X}^{*}(\mathbb{T}, E)\right\}, \\
& Z_{r}:=T_{r} \cap Z_{G} .
\end{aligned}
$$

Note that $Z_{r}$ is well defined independent of the choice of $T$.

In the rest of this paper, $E$ will denote a tamely ramified finite extension of $F$ unless otherwise stated.

\subsection{Cocenters.}

1.2.1. For $s \in \tilde{\mathbb{R}}_{\geq 0}$, let $\mathcal{H}\left(G, G_{x, s}\right)$ be the space of compactly supported, $G_{x, s} \times$ $G_{x, s}$-invariant $\mathbb{Z}\left[\frac{1}{p}\right]$-valued functions on $G$ and let $C_{c}\left(G / G_{x, s}\right)$ be the space of compactly supported, right $G_{x, s}$-invariant $\mathbb{Z}\left[\frac{1}{p}\right]$-valued functions on $G$. Note that for any $g \in G$ and $x \in \mathcal{B}(G)$, we have

$$
\mathbb{1}_{g G_{x, s}} \equiv \frac{\mu_{G}\left(G_{x, s}\right)}{\mu_{G}\left(G_{x, s} g G_{x, s}\right)} \mathbb{1}_{G_{x, s} g G_{x, s}} \bmod [\mathcal{H}, \mathcal{H}]
$$

Thus $\mathcal{H}\left(G, G_{x, s}\right)$ and $C_{c}\left(G / G_{x, s}\right)$ have the same image in $\overline{\mathcal{H}}$. We denote by $\overline{\mathcal{H}}_{s}$ the image of $\mathcal{H}_{s}=\sum_{x \in \mathcal{B}(G)} C_{c}\left(G / G_{x, s}\right)$ in $\overline{\mathcal{H}}$. Then $\overline{\mathcal{H}}=\lim _{s} \overline{\mathcal{H}}_{s}$.

We set $\overline{\mathcal{H}}_{s}^{\text {rig }}=\overline{\mathcal{H}}^{\text {rig }} \cap \overline{\mathcal{H}}_{s}$. Then $\overline{\mathcal{H}}^{\text {rig }}=\lim _{s} \overline{\mathcal{H}}_{s}^{\text {rig }}$. 
A $G$-domain, by definition, is an open and closed subset of $G$ that is stable under the conjugation action of $G$. We have the following simple facts about the cocenter $\overline{\mathcal{H}}$.

Lemma 1.2.2. Let $X$ be a $G$-domain and let $\mathcal{H}(X)$ be the $\mathbb{Z}\left[\frac{1}{p}\right]$-submodule of $\mathcal{H}$ consisting of functions supported in $X$. Then $\mathcal{H}(X) \cap[\mathcal{H}, \mathcal{H}]$ is spanned by $f-{ }^{g} f$ for $f \in \mathcal{H}(X)$ and $g \in G$.

Proof. Let $f \in \mathcal{H}(X) \cap[\mathcal{H}, \mathcal{H}]$. By [12, Proposition 1.1], $f=\sum_{i}\left(f_{i}-{ }^{g_{i}} f_{i}\right)$, where $f_{i} \in \mathcal{H}$ and $g_{i} \in G$. Since $X$ is a $G$-domain, $\left.f_{i}\right|_{X} \in \mathcal{H}(X)$ and $\left.\left({ }^{g_{i}} f_{i}\right)\right|_{X}={ }^{g_{i}}\left(\left.f_{i}\right|_{X}\right)$ for any $i$. Thus $f=\sum_{i}\left(\left.f_{i}\right|_{X}-\left.{ }^{g_{i}} f_{i}\right|_{X}\right)$.

Lemma 1.2.3. Let $\left\{X_{\alpha}\right\}_{\alpha \in I}$ be a family of $G$-domains in $G$ such that $X_{\alpha} \cap X_{\alpha^{\prime}}=\emptyset$ for any $\alpha \neq \alpha^{\prime}$. Then $\sum_{\alpha \in I} \overline{\mathcal{H}}\left(X_{\alpha}\right) \subset \overline{\mathcal{H}}$ is a direct sum. Here $\overline{\mathcal{H}}\left(X_{\alpha}\right)$ is the image of $\mathcal{H}\left(X_{\alpha}\right)$ in $\overline{\mathcal{H}}$.

If moreover $G=\bigsqcup_{\alpha \in I} X_{\alpha}$, then $\overline{\mathcal{H}}=\bigoplus_{\alpha} \overline{\mathcal{H}}\left(X_{\alpha}\right)$.

Proof. Let $f_{\alpha} \in \mathcal{H}\left(X_{\alpha}\right), \alpha \in I$ such that $\Gamma:=\left\{\alpha ; f_{\alpha} \neq 0\right\}$ is a finite set. Suppose that $\sum_{\alpha \in \Gamma} f_{\alpha} \in[\mathcal{H}, \mathcal{H}]$. Then by [12, Proposition 1.1], there exists finitely many pairs $\left(f_{i}, x_{i}\right) \in \mathcal{H} \times G$ such that

$$
\sum_{\alpha \in \Gamma} f_{\alpha}=\sum_{i}\left(f_{i}-{ }^{x_{i}} f_{i}\right)
$$

Restricting both sides of (a) to $X_{\alpha}$, we have $f_{\alpha}=\sum_{i}\left(\left.f_{i}\right|_{X_{\alpha}}-\left.\left({ }^{x_{i}} f_{i}\right)\right|_{X_{\alpha}}\right)$. Since $X_{\alpha}$ is a $G$-domain, we have $\left.f_{i}\right|_{X_{\alpha}} \in \mathcal{H}$ and $\left.\left({ }^{x_{i}} f_{i}\right)\right|_{X_{\alpha}}={ }^{x_{i}}\left(\left.f_{i}\right|_{X_{\alpha}}\right)$.

Thus $f_{\alpha}=\sum_{i}\left(\left.f_{i}\right|_{X_{\alpha}}-{ }^{x_{i}}\left(\left.f_{i}\right|_{X_{\alpha}}\right)\right) \in[\mathcal{H}, \mathcal{H}]$. Therefore the image of $f_{\alpha}$ in $\overline{\mathcal{H}}$ is zero and $\sum_{\alpha} \overline{\mathcal{H}}\left(X_{\alpha}\right) \subset \overline{\mathcal{H}}$ is a direct sum.

If moreover $G=\sqcup_{\alpha} X_{\alpha}$, then for any $f \in \mathcal{H}, f=\left.\sum_{\alpha} f\right|_{X_{\alpha}} \in \sum_{\alpha} \mathcal{H}\left(X_{\alpha}\right)$. Hence $\overline{\mathcal{H}}=\sum \overline{\mathcal{H}}\left(X_{\alpha}\right)$. By what we proved above, this is a direct sum.

\section{Semisimple elements And DeComposition of $G^{\text {rig }}$}

From now on, let $r$ be a positive real number.

2.1. Depth functions and good elements. The following definitions in Definitions 2.1.1 and 2.1.2 coincide with those in [3] when $N=Z(G)$.

Definition 2.1.1. Write $Z:=Z(G)$. For $x \in \mathcal{B}(G)$, define the depth-mod-center function

$$
d^{\mathbb{G}}(x,): Z \operatorname{Stab}_{G}(x) \rightarrow \mathbb{R} \sqcup\{\infty\},
$$

such that

$$
d^{\mathbb{G}}(x, g)=\left\{\begin{array}{cl}
0 & \text { if } g \in Z \operatorname{Stab}_{G}(x) \backslash Z G_{x, 0^{+}} \\
\max \left\{s \mid z g \in G_{x, s} \text { for some } z \in Z\right\} & \text { if } g \in Z G_{x, 0^{+}} \backslash Z \\
\infty & \text { if } g \in Z
\end{array}\right.
$$

Define also

$$
d^{\mathbb{G}}(g)=\max \left\{d^{\mathbb{G}}(x, g) \mid x \in \mathcal{B}(G), g \in \operatorname{Stab}_{G}(x)\right\} .
$$

We simply write $d$ for $d^{\mathbb{G}}$ if there is no confusion. 
We observe the following:

(1) If $g \in Z G_{x, 0^{+}} \backslash Z, d(x, g)$ is the unique value $t$ so that $g \in Z G_{x, t} \backslash Z G_{x, t^{+}}$. In most applications, it is possible to assume that $g \in G_{x, t} \backslash Z G_{x, t^{+}}$without loss of generality. In this case, we call $g$ noncentral $\bmod G_{x, t^{+}}$.

Likewise, when $d(g)=0$, one may assume that $g \in \operatorname{Stab}_{G}(x) \backslash Z G_{x, 0^{+}}$ in most cases. Note that $\operatorname{Stab}_{G}(x)$ is compact since $\mathcal{B}(G)$ is an extended building. Again, we say $g$ is noncentral $\bmod G_{x, 0^{+}}$if $g \in \operatorname{Stab}_{G}(x) \backslash Z G_{x, 0^{+}}$.

(2) $d(y, g) \leq d(g)$ for any $y \in \mathcal{B}(G), g \in \operatorname{Stab}_{G}(y)$.

(3) If $g \in Z G_{0} \backslash Z, d(g)$ is the unique nonnegative real number $t$ such that $g \in Z G_{t} \backslash Z G_{t^{+}}$.

(4) $d(x, g)=d\left(x, g^{\prime}\right)$ for all $g^{\prime} \in g G_{x, t^{+}}$where $d(x, g)=t$.

(5) $d(g)=\infty$ if and only if $g \in Z \mathcal{U} \cap G$. This follows from [2, Lemma 3.7.13].

(6) Let $g \in G^{\text {rig }}$. If $g=\gamma u$ is the Jordan decomposition of $g$ with $\gamma \in G^{s s}$ and $u \in \mathcal{U}$, we have $d(g)=d(\gamma)$.

This follows from the proof of [2, Lemma 3.7.18]. Although the lemma assumes the characteristic of $F$ being zero, the proof requires only $\gamma$ being rational and the residue field of $F$ being finite. We thank Jeff Adler for clarification.

Definition 2.1.2 (cf. Definition 6.1 of $[3]$ ). For $\gamma \in G^{\text {rig }}, \gamma$ is a $\mathbb{G}$-good mod center element if there is a maximal $F$-torus $\mathbb{T}$ which splits over a tamely ramified extension $E$ such that one of the the following holds:

(1) $\gamma \in Z T^{c} \backslash Z T_{0^{+}}$and the image of $\gamma$ in $\bar{G}$ is absolutely semisimple (see [11] or [3, Definition 4.11] for a definition), where $T^{c}$ is the set of compact elements in $T$.

(2) There is $t>0$ so that $\gamma \in Z T_{t} \backslash Z T_{t^{+}}$with $\nu(\alpha(\gamma)-1)=t$ or $\alpha(\gamma)=1$ for any $\alpha \in \Phi(\mathbb{T}, \mathbb{G}, E)$.

(3) $\gamma \in Z$.

We will simply say $\gamma$ is $\mathbb{G}$-good of depth $t$ if $\gamma$ is $\mathbb{G}$-good mod center and if either $d(\gamma)=0$ and $\gamma \in T^{c}$, or $d(\gamma)=t>0$ and $\gamma \in T_{t}$.

Remarks 2.1.3. Keeping the situation as in the above definition, we observe the following:

(1) The depth of a good mod center element $\gamma$ is given as follows:

$$
d(\gamma)= \begin{cases}0 & \text { in case }(1) \\ t & \text { in case }(2), \\ \infty & \text { in case }(3)\end{cases}
$$

(2) If $\gamma \in T \backslash Z$ is a good mod center element of depth $t>0$ (resp., 0 ), $\gamma=z \gamma_{t}$ for some $z \in Z$ and a good element $\gamma_{t} \in T_{t} \backslash T_{t^{+}}$(resp., $\gamma \in T^{c} \backslash T_{0^{+}}$).

(3) Let $\gamma \in G^{s s}$ and $\mathbb{G}^{\prime}=C_{\mathbb{G}}(\gamma)$. Let $d^{\mathbb{G}^{\prime}}$ be the depth function defined on $G^{\text {rig }}$ as in Definition 2.1.1. In general $d^{\mathbb{G}^{\prime}} \neq d^{\mathbb{G}}$ on $G^{\text {rig }}$. However, if $g \in G^{\prime \text { rig }} \backslash Z\left(G^{\prime}\right)$ is $\mathbb{G}$-good, it is also $\mathbb{G}^{\prime}$-good and $d^{\mathbb{G}^{\prime}}(g)=d^{\mathbb{G}}(g)$.

2.2. Hypotheses. We collect here some assumptions that we need in this paper. We will be clear when each hypothesis is used. Many of them are due to results we use from [3] and 9. Rather than repeating the statements of the hypotheses, we refer the reader directly to [3] and $[9]$. 
Hypotheses (A)-(D). These are Hypotheses (A)-(D) in [3, §2].

Hypotheses (DB). These are the hypotheses in $\S \S 2.1$ and 4.3 in [9].

Hypothesis 1. The Jordan decomposition is defined over $F$, i.e., for any $g \in G=$ $\mathbb{G}(F)$ and Jordan decomposition $g=$ su of $g$ with $s, u \in \mathbb{G}(\bar{F})$, we have $s, u \in G$.

Hypothesis 2. For any $\gamma \in G^{s s}$, all the unipotent elements in $C_{G}(\gamma)$ are contained in $C_{G}(\gamma)^{\circ}$.

Hypothesis 3. Any torus in $\mathbb{G}$ splits over a finite tamely ramified extension of $F$.

Hypothesis 4 (Definition 6.3 of [3]). For any torus $\mathbb{S} \subset \mathbb{G}$ which splits over a tamely ramified extension $E$, and $r>0$, every nontrivial coset in $S_{r} / S_{r+}$ contains a good element.

Hypothesis 5. For any $g \in G^{\mathrm{rig}}$, the orbital integral $O_{g}$ converges over $\mathbb{C}$.

Hypothesis 1 holds if $F$ is of characteristic zero, or if $p>\operatorname{rank}_{s s}(G)+1$. But it fails when $F$ is of positive characteristic and $p$ is small. See [20, Proposition 48 and Remark 49]. Hypothesis 2 automatically holds if $F$ is of characteristic zero. If $F$ is of characteristic $p$, then it holds when $p$ is large but fails for some small $p$. For example, when $p=2$ and $C_{G}(\gamma)$ has two connected components, then any elements in $C_{G}(\gamma) \backslash C_{G}(\gamma)^{\circ}$ of order 2 is unipotent. Hypotheses 3 and 4 hold when $\mathbb{G}$ splits over a tamely ramified extension and $p$ does not divide the order of the Weyl group of $\mathbb{G}$ (see [10]). Hypothesis 5 holds if $F$ is of characteristic zero (see [24]), and holds under some mild assumptions on $G$ and on $p$ if $F$ is of positive characteristic (see [20, Theorem 61] for the precise statement).

2.3. Good elements and $\mathcal{B}(G)$. Many results here can be found in 3 . For the Lie algebra versions, we refer to $17-19$.

In the following four lemmas and a corollary, we let $\gamma \in T$ be a $\mathbb{G}$-good mod center element of depth $t \geq 0$. We also let $\mathbb{G}^{\prime}:=C_{\mathbb{G}}(\gamma)$.

Lemma 2.3.1. Suppose Hypotheses (A) and (B) hold. Define $\mathcal{B}(\gamma)$ as follows:

$$
\mathcal{B}(\gamma):= \begin{cases}\left\{x \in \mathcal{B}(G) \mid \gamma \in Z \operatorname{Stab}_{G}(x)\right\} & \text { if } t=0, \infty, \\ \{x \in \mathcal{B}(G) \mid d(x, \gamma)=d(\gamma)\} & \text { if } t>0 .\end{cases}
$$

Then, we have $\mathcal{B}(\gamma)=\mathcal{B}\left(\mathbb{G}^{\prime}, F\right)$.

Proof. If $\gamma \in Z$, clearly $\mathcal{B}(\gamma)=\mathcal{B}(G)$. Otherwise, without loss of generality, we may assume that $\gamma$ is $\mathbb{G}$-good of depth $t$. Then, the lemma follows from 3 , Lemma 7.6].

Lemma 2.3.2. Suppose Hypothesis (A) holds. Let $x \in \mathcal{B}\left(G^{\prime}\right)$. Then, for $0 \leq t<s$ and $u \in G_{x, t}^{\prime} \cap G_{t^{+}}^{\prime}$, we have $G_{x, s-t}\left(\gamma u G_{x, s}^{\prime}\right)=\gamma u G_{x, s}$.

Proof. One may assume that $u$ is semisimple since $G^{\prime s s} \cap u G_{x, s}^{\prime} \cap G_{t^{+}}^{\prime} \neq \emptyset$. Then, this follows from [3, Corollary 7.5].

Lemma 2.3.3. Suppose Hypothesis (C) holds. If $g \in G$ is such that ${ }^{g}\left(\gamma G_{t^{+}}^{\prime}\right) \cap$ $\left(\gamma G_{t^{+}}^{\prime}\right) \neq \emptyset$, then $g \in G^{\prime}=\mathbb{G}^{\prime}(F)$.

Proof. Without loss of generality, we may assume $\gamma$ is $\mathbb{G}$-good. Then, this is 3 , Lemma 7.1]. 
Lemma 2.3.4. Suppose Hypotheses (A) and (B) hold. Let $x \in \mathcal{B}(G) \backslash \mathcal{B}\left(G^{\prime}\right)$ and $u^{\prime} \in G_{d(\gamma)^{+}}^{\prime}$.

(1) If $t=0, \gamma u^{\prime} \notin Z \operatorname{Stab}_{G}(x)$.

(2) If $t>0$, then either $\gamma u^{\prime} \notin Z \operatorname{Stab}_{G}(x)$ or $d\left(x, \gamma u^{\prime}\right)<d(\gamma)$.

In particular, $\left\{x \in \mathcal{B}(G) \mid d\left(x, \gamma u^{\prime}\right)=d\left(\gamma u^{\prime}\right)\right\} \subset \mathcal{B}\left(G^{\prime}\right)$.

Proof. When $u^{\prime}=1$, this is [3, Lemma 7.6].

If $\gamma \in Z$, the statement is empty. We may assume that $\gamma$ is a $\mathbb{G}$-good element.

(1) Suppose $\gamma u^{\prime} \in \operatorname{Stab}_{G}(x)$. Then, we have $\gamma^{-1} x=u^{\prime} x$ and $B:=\left\{\gamma^{-p^{n}} x \mid\right.$ $\left.n \in \mathbb{Z}_{\geq 0}\right\}=\left\{u^{\prime p^{n}} x \mid n \in \mathbb{Z}_{\geq 0}\right\} \subset \mathcal{B}(G)$. Since $u^{\prime p^{n}} \rightarrow 1$ as $n \rightarrow \infty$, the set $B$ is finite and $x=u^{\prime p^{n}} x=\gamma^{-\bar{p}^{n}} x$ for sufficiently large $n$. On the other hand, since $\gamma$ is absolutely semisimple and the order of $\gamma$ is relatively prime to $p$, there is an $n_{\circ} \in \mathbb{Z}_{>0}$ such that $\gamma^{-p^{n_{\circ} \ell}}=\gamma^{-1}$ for any $\ell \in \mathbb{Z}_{>0}$.

Hence $\gamma x=x$, that is, $\gamma \in \operatorname{Stab}_{G}(x)$, which is a contradiction to Lemma 2.3.1.

(2) As in [3, Lemma 7.6], we may assume that $\gamma$ is split. Write $t=d(\gamma)$. Write $\gamma^{\prime}$ for $\gamma u^{\prime}$, and define

$$
\mathcal{B}\left(\gamma^{\prime}\right):=\left\{x \in \mathcal{B}(\mathbb{G}, F) \mid d\left(x, \gamma^{\prime}\right) \geq t\right\} .
$$

Note that $\mathcal{B}\left(\gamma^{\prime}\right)$ is convex and is a union of closures of chambers.

It is enough to show that $\mathcal{B}\left(\gamma^{\prime}\right) \subset \mathcal{B}\left(\mathbb{G}^{\prime}, F\right)$.

Suppose first that $\gamma$ is split and $\mathbb{G}^{\prime}$ is an $F$-Levi subgroup of $\mathbb{G}$. Then, $t \in \mathbb{N}$. Let $\mathbb{P}$ be a $k$-parabolic subgroup of $\mathbb{G}$ having Levi decomposition $\mathbb{P}=\mathbb{G}^{\prime} \mathbb{N}$. Let $\overline{\mathbb{P}}$ be the parabolic subgroup opposite to $\mathbb{P}$ with respect to this Levi decomposition $\mathbb{P}=\mathbb{G}^{\prime} \mathbb{N}$. Let $\overline{\mathbb{N}}$ be the unipotent radical of $\overline{\mathbb{P}}$. Now, assume $\mathcal{B}\left(\gamma^{\prime}\right) \backslash \mathcal{B}\left(\mathbb{G}^{\prime}, F\right) \neq \emptyset$, and let $D$ be a chamber in $\mathcal{B}\left(\gamma^{\prime}\right) \backslash \mathcal{B}\left(\mathbb{G}^{\prime}, F\right)$. From the convexity of $\mathcal{B}\left(\gamma^{\prime}\right)$, we may assume that $\bar{D}$ shares a facet $F$ of codimension one with $\mathcal{B}\left(\gamma^{\prime}\right) \cap \mathcal{B}\left(\mathbb{G}^{\prime}, F\right)$. Choose $y \in F$. From [2, (2.4.1)], there is $u \in G_{y, 0} \cap N$ such that $u D \subset \mathcal{B}\left(\mathbb{G}^{\prime}, F\right)$. Then for $x \in D, u x \in u D \subset \mathcal{B}\left(\mathbb{G}^{\prime}, F\right)$. Since $u x \in \mathcal{B}\left(\mathbb{G}^{\prime}, F\right)\left[22\right.$ and $u D$ is maximal, $G_{u x, t}$ has an Iwahori decomposition with respect to $(P, N)$, that is,

$$
G_{u x, t}=N_{u x, t} \cdot G_{u x, t}^{\prime} \cdot \bar{N}_{u x, t},
$$

where $N_{u x, t}=G_{u x, t} \cap N$ and $\bar{N}_{u x, t}=G_{u x, t} \cap \bar{N}$. From this and the fact that $u \in N$, we can decompose ${ }^{u} \gamma^{\prime}$ as

$$
{ }^{u} \gamma^{\prime} \equiv \gamma^{\prime-1}\left({ }^{u} \gamma^{\prime}\right) \gamma^{\prime} \in G_{y, t} / G_{y, t^{+}},
$$

where $\gamma^{\prime} \in G^{\prime} \cap G_{y, t}=G_{y, t}^{\prime}$ and $\gamma^{\prime-1 u} \gamma^{\prime} \in N_{y, t}$. Since $G_{y, t^{+}} \subset G_{u x, t^{+}} \subset G_{u x, t} \subset$ $G_{y, t}$, comparing $(\dagger)$ and $(\ddagger)$, we have $\gamma^{\prime} \in G^{\prime} \cap G_{u x, t}=G_{u x, t}^{\prime}$ and $\gamma^{\prime-1 u} \gamma^{\prime} \in N_{u x, t}$. Moreover,
(i) $u^{\prime} \in G_{u x, t^{+}}$,
(ii) ${ }^{u} \gamma^{\prime} \in \gamma^{\prime} G_{u x, t^{+}}=\gamma G_{u x, t^{+}}$.

(i) follows from the fact that $u D$ is a chamber and $u^{\prime} \in G_{u x, t} \cap G_{t^{+}}^{\prime}($ recall $t \in \mathbb{N}$ ). (ii) follows from (i) and the fact that $u D$ is a chamber and thus $N_{u x, t}=N_{u x, t^{+}}$. Then from Lemma 2.3.2, there is a $k \in G_{u x, 0^{+}}$and a $u^{\prime \prime} \in G_{u x, t^{+}}^{\prime}=G^{\prime} \cap G_{u x, t^{+}}$such that ${ }^{u} \gamma^{\prime}={ }^{k}\left(\gamma u^{\prime \prime}\right)$. By Lemma 2.3.3, $u \in k \cdot G^{\prime} \subset G_{u x, 0^{+}} G^{\prime}$. Since $u x \in \mathcal{B}\left(\mathbb{G}^{\prime}, F\right)$, we have $G_{u x, 0^{+}}=\left(N \cap G_{u x, 0^{+}}\right) \cdot\left(G^{\prime} \cap G_{u x, 0^{+}}\right) \cdot\left(\bar{N} \cap G_{u x, 0^{+}}\right)$[22, (4.2)]. So, we can conclude that $u \in G_{u x, 0^{+}}$and $u(u x)=u x=x$. Then $x \in \mathcal{B}\left(\mathbb{G}^{\prime}, F\right)$, which is a contradiction.

The following is a corollary of the above lemmas. 
Corollary 2.3.5. Suppose Hypotheses (A) and (B) hold. Let $u^{\prime} \in G_{d(\gamma)^{+}}^{\prime}$. Let $x \in \mathcal{B}(G)$.

(1) If $t=0$ and $\gamma u^{\prime} \in Z \operatorname{Stab}_{G}(x)$, both $\gamma$ and $u^{\prime}$ are also in $Z \operatorname{Stab}_{G}(x)$.

(2) If $t>0$ and $\gamma u^{\prime} \in G_{x, t}$, both $\gamma$ and $u^{\prime}$ are also in $Z G_{x, t}$.

Proof. This follows from Lemmas 2.3.1 and 2.3.4.

\subsection{Good products.}

Lemma 2.4.1. Suppose Hypothesis (C) holds. Let $\mathbb{T}$ be a maximal F-torus in $\mathbb{G}$ which splits over a tamely ramified Galois extension $E$. Let $\gamma_{1}, \cdots, \gamma_{n} \in T$ be $\mathbb{G}$-good elements of depth $b=b_{1}, \cdots, b_{n}$, respectively. Let $\gamma=\gamma_{z} \gamma_{1}$ with $\gamma_{z} \in Z$. Let $\mathbb{H}^{0}:=\mathbb{G}$ and $\mathbb{H}^{i}:=C_{\mathbb{H}^{i-1}}\left(\gamma_{i}\right)$.

(1) Let $\gamma^{\prime} \in \gamma H_{b^{+}}^{1}$. Then, $C_{G}\left(\gamma^{\prime}\right) \subset H^{1}$. If $\gamma^{\prime}$ is also $\mathbb{G}$-good mod center of depth $b$, then $C_{G}\left(\gamma^{\prime}\right)=H^{1}$.

(2) Suppose $b_{1}<b_{2}<\cdots<b_{n}$. Fix $i \in\{0,1, \cdots, n\}$ and $\gamma^{i}=\gamma_{z} \gamma_{1} \cdots \gamma_{i}$. Let $\gamma^{\prime}, \gamma^{\prime \prime} \in \gamma^{i} H_{b_{i}^{+}}^{i}$. If ${ }^{g} \gamma^{\prime}=\gamma^{\prime \prime}$ for some $g \in G$, then $g \in H^{i}$.

(3) $H^{i}=C_{H^{i-1}}\left(\gamma^{i}\right)=C_{G}\left(\gamma^{i}\right)$. In particular, $C_{G}\left(\gamma^{n}\right) \subset H^{i}, i=1, \cdots, n$.

Proof. (1) If $\gamma_{z}=1$, the first statement is Lemma2.3.3. Since $\gamma_{z} \in Z$, the statement remains valid for this case. For the second statement, since $\gamma^{\prime}$ is also good of depth $b, H^{1} \subset C_{G}\left(\gamma^{\prime}\right)$. Combining this with the first statement, the second statement follows.

(2) We use induction on $i$. Since $\Phi\left(\mathbb{T}, \mathbb{H}^{i}, E\right) \subset \Phi(\mathbb{T}, \mathbb{G}, E)$ and $\gamma_{i} \in \mathbb{T}_{b_{i}}$, each $\gamma_{i}, i=1, \cdots, k$ is also $\mathbb{H}^{i-1}$-good. When $i=1$, it is (1). Assume the statement is true for $i-1 \geq 1$. Note that $\gamma^{\prime}, \gamma^{\prime \prime} \in \gamma^{i} H_{b_{i}^{+}}^{i}$. Suppose ${ }^{g} \gamma^{\prime}=\gamma^{\prime \prime}$ for some $g \in G$. Since $\gamma^{\prime}, \gamma^{\prime \prime} \in \gamma^{i-1} H_{b_{i-1}^{+}}^{i-1}$, we have $g \in H^{i-1}$ by the induction hypothesis and ${ }^{g}\left(\gamma^{\prime}\left(\gamma^{i-1}\right)^{-1}\right)=\gamma^{\prime \prime}\left(\gamma^{i-1}\right)^{-1}$. Since $\gamma^{\prime}\left(\gamma^{i-1}\right)^{-1}, \gamma^{\prime \prime}\left(\gamma^{i-1}\right)^{-1} \in \gamma_{i} H_{b_{i}^{+}}^{i}$ and $\gamma_{i}$ is $\mathbb{H}^{i-1}$-good and $\gamma^{i-1} \in Z\left(H^{i-1}\right), g \in H^{i}=C_{H^{i-1}}\left(\gamma_{i}\right)=C_{H^{i-1}}\left(\gamma^{i}\right)$ by $(1)$.

(3) The first equality follows since $\gamma^{i-1} \in Z\left(H^{i-1}\right)$ and $\gamma^{i}=\gamma^{i-1} \gamma_{i}$. To prove the second equality, we use an induction. If $i=1$, it is trivial. Suppose $i-1 \geq 1$. The inclusion $C_{H^{i-1}}\left(\gamma^{i}\right) \subset C_{G}\left(\gamma^{i}\right)$ is obvious. If $g \in C_{G}\left(\gamma^{i}\right)$, we have ${ }^{g} \gamma^{i}, \gamma^{i} \in$ $\gamma^{i} H_{b_{i}^{+}}^{i} \subset \gamma^{i-1} H_{b_{i-1}^{+}}^{i-1}$. Then, $g \in H^{i-1}$ by (2). Hence, $C_{G}\left(\gamma^{i}\right) \subset C_{H^{i-1}}\left(\gamma^{i}\right)$.

Lemma 2.4.2. Let $\mathbb{T}$ be a maximal $F$-torus in $\mathbb{G}$ which splits over a tamely ramified Galois extension E. Let $\gamma_{1}, \gamma_{2} \in T$ be $\mathbb{G}$-good mod center elements of depth $b_{1}, b_{2}$, respectively. Let $\mathbb{H}^{i}=C_{\mathbb{H}}\left(\gamma_{i}\right), i=1,2$. Suppose $b_{1}<b_{2}$ and $\gamma_{2} \in Z\left(H^{1}\right)$. Then, $\gamma_{1} \gamma_{2}$ is also a $G$-good element of depth $b_{1}$.

Proof. Write $\gamma=\gamma_{1} \gamma_{2}$. Let $\Phi:=\Phi(\mathbb{T}, \mathbb{G}, E)$ be the set of $E$-rational $\mathbb{T}$-roots in $\mathbb{G}$. Let $\alpha \in \Phi$. Since $\mathbb{H}^{1} \subset C_{\mathbb{G}}\left(\gamma_{2}\right), \alpha\left(\gamma_{1}\right)=1$ implies $\alpha\left(\gamma_{2}\right)=1$, thus $\alpha\left(\gamma_{1} \gamma_{2}\right)=1$. If $\alpha\left(\gamma_{1}\right) \neq 1$, since $\alpha\left(\gamma_{1} \gamma_{2}\right)-1=\alpha\left(\gamma_{1}\right) \alpha\left(\gamma_{2}\right)-\alpha\left(\gamma_{2}\right)+\alpha\left(\gamma_{2}\right)-1, \nu\left(\alpha\left(\gamma_{2}\right)\right)=0$ and $b_{1}=\nu\left(\alpha\left(\gamma_{1}\right)-1\right)<b_{2} \leq \nu\left(\alpha\left(\gamma_{2}\right)-1\right)$, we have $\nu(\alpha(\gamma)-1)=\min \left(\nu\left(\alpha\left(\gamma_{1}\right)-\right.\right.$ $\left.1), \nu\left(\alpha\left(\gamma_{2}\right)-1\right)\right)=b_{1}$. Hence, $\gamma$ is $\mathbb{G}$-good mod center of depth $b_{1}$.

Proposition 2.4.3. Suppose Hypotheses (C) and 4 hold. Let $\mathbb{T}$ be an E-split torus and $\gamma \in Z T^{c}$. Then $\gamma$ is a product of good elements mod $r^{+}$with decreasing 
centralizers in the following sense:

(1) $\gamma=\gamma_{z} \gamma_{1} \cdots \gamma_{k} \gamma_{r^{+}}$where $\gamma_{z} \in Z$ and each $\gamma_{i}$ is $\mathbb{G}$-good of depth $b_{i}$ with $b_{1}<b_{2}<\cdots<b_{k} \leq r$ and $\gamma_{r^{+}} \subset T_{r^{+}}$, that is, $d\left(\gamma_{r^{+}}\right)>r$.

(2) $H^{1} \supsetneq H^{2} \supsetneq \cdots \supsetneq H^{k}$ where $H^{i}=C_{G}\left(\gamma_{1} \cdots \gamma_{i}\right)$.

Proof. If $d(\gamma)>r, \gamma=\gamma_{z} \cdot \gamma_{r^{+}}$for some $\gamma_{z} \in Z$ and $\gamma_{r^{+}} \in T_{r^{+}}$. Now, we assume that $d(\gamma)=a_{1} \leq r$.

We first assume that $\gamma \in T_{a_{1}}$, that is, $\gamma$ is noncentral $\bmod T_{a_{1}^{+}}$. Then, $\gamma T_{a_{1}^{+}}$ contains a good element, say, $\tilde{\gamma}_{a_{1}}$ of depth $a_{1}$ by Hypothesis 4 if $a_{1}>0$ and by Corollary 2.35 of [26] if $a_{1}=0$. Write $\gamma=\tilde{\gamma}_{a_{1}}\left(\gamma \tilde{\gamma}_{a_{1}}^{-1}\right)$ with $a_{2}=d\left(\gamma \tilde{\gamma}_{a_{1}}^{-1}\right)>d(\gamma)$. We can choose $\tilde{\gamma}_{a_{1}}$ so that $\gamma \tilde{\gamma}_{a_{1}}^{-1} \in T_{a_{2}}$ by multiplying $\tilde{\gamma}_{a_{1}}$ with a central element if necessary (note that $a_{2}=d\left(\gamma \tilde{\gamma}_{a_{1}}^{-1}\right)$ implies only $\left.\gamma \tilde{\gamma}_{a_{1}}^{-1} \in Z T_{a_{2}}\right)$. Applying the above process for $\gamma \tilde{\gamma}_{a_{1}}^{-1}$, we find a $\mathbb{G}$-good element $\tilde{\gamma}_{a_{2}} \in \gamma \tilde{\gamma}_{a_{1}}^{-1} T_{a_{2}^{+}}$such that $\gamma=$ $\tilde{\gamma}_{a_{1}} \tilde{\gamma}_{a_{2}}\left(\gamma\left(\tilde{\gamma}_{a_{1}} \tilde{\gamma}_{a_{2}}\right)^{-1}\right)$ and $a_{3}=d\left(\gamma\left(\tilde{\gamma}_{a_{1}} \tilde{\gamma}_{a_{2}}\right)^{-1}\right)<d\left(\gamma \tilde{\gamma}_{a_{1}}^{-1}\right)$. Repeatedly, we have

$$
\gamma=\tilde{\gamma}_{a_{1}} \tilde{\gamma}_{a_{2}} \ldots \tilde{\gamma}_{a_{m}} \tilde{\gamma}_{r^{+}},
$$

where $\tilde{\gamma}_{a_{i}}$ is a $\mathbb{G}$-good element of depth $a_{i}$ with $a_{1}<a_{2}<\cdots<a_{m} \leq r$ and $d\left(\tilde{\gamma}_{r^{+}}\right)>r$. This procedure is finite because $d\left(T_{0}\right) \subset \frac{1}{e(E / F)} \mathbb{Z}$. Put $a_{m+1}:=r^{+}$ and $\tilde{\gamma}_{a_{m+1}}=\tilde{\gamma}_{r^{+}}$.

Set $S:=\left\{a_{1}, a_{2}, \cdots, a_{m+1}\right\}$, and for $a, b \in \widetilde{\mathbb{R}}$, set $\tilde{\gamma}_{a, b}:=\prod_{a \leq a_{j}<b} \tilde{\gamma}_{a_{j}}$. We find a subsequence $b_{1}<b_{2}<\cdots<b_{n}<b_{n+1}$ of $S$ as follows: let $b_{1}:=a_{1}$ and $\mathbb{H}^{1}:=C_{\mathbb{G}}\left(\tilde{\gamma}_{a_{1}}\right)$. Let $b_{2}$ be the maximal element in $\left\{a_{2}, \cdots, a_{m+1}\right\}$ with the property that if $a_{j}<b_{2}$, then $\tilde{\gamma}_{a_{j}} \in Z\left(H^{1}\right)$. Note that $\mathbb{H}^{1}=C_{\mathbb{G}}\left(\tilde{\gamma}_{b_{1}, b_{2}}\right)$. Let $\mathbb{H}^{2}:=C_{\mathbb{H}^{1}}\left(\tilde{\gamma}_{b_{2}}\right)$. Then $\mathbb{H}^{1} \supsetneq \mathbb{H}^{2}$. Let $\gamma_{1}:=\tilde{\gamma}_{b_{1}, b_{2}}$. Inductively, suppose $b_{i}, \mathbb{H}^{i}$ and $\gamma_{i-1}$ are defined for $i \geq 2$. Let $b_{i+1}$ be the maximal element in $\left\{a_{j} \in S \mid a_{j}>b_{i}\right\}$ with the property that for any $a_{j}<b_{i+1}, \tilde{\gamma}_{a_{j}} \in Z\left(H^{i}\right)$. Let $\mathbb{H}^{i+1}:=C_{\mathbb{H}^{i}}\left(\tilde{\gamma}_{b_{i+1}}\right)$ and $\gamma_{i}=\tilde{\gamma}_{b_{i}, b_{i+1}}$. We repeat the process until $b_{n+1}=a_{m+1}=r^{+}$.

Then each $\gamma_{i}$ is also a $G$-good element of depth $b_{i}$, and we have $\mathbb{H}^{i}=C_{\mathbb{H}^{i-1}}\left(\tilde{\gamma}_{b_{i}}\right)=$ $C_{\mathbb{H}^{i-1}}\left(\gamma_{i}\right), i=1, \cdots, n$ by Lemma 2.4.1(1) . Now, one can easily check

$$
\gamma=\gamma_{1} \gamma_{2} \cdots \gamma_{n} \gamma_{r+}
$$

satisfies the required properties.

Now suppose $\gamma \in Z T_{a_{1}}$. Then one can write $\gamma=\gamma_{z} \gamma^{\prime}$ with $\gamma_{z} \in Z$ and $\gamma^{\prime} \in T_{a_{1}}$ noncentral mod $T_{a_{1}^{+}}$. Write $\gamma^{\prime}=\gamma_{1} \cdots \gamma_{n} \gamma_{r+}$ as in (*). Then, $\gamma=\gamma_{z} \gamma_{1} \gamma_{2} \cdots \gamma_{n} \gamma_{r^{+}}$ satisfying the required properties.

\section{Definition 2.4.4.}

(1) We call the expression $\gamma_{z} \cdot \gamma_{1} \cdots \gamma_{k} \gamma_{r^{+}}$of $\gamma$ in Proposition 2.4 .3 a good product of $\gamma \bmod r^{+}$. That is, $\gamma=\gamma_{z} \cdot \gamma_{1} \cdots \gamma_{k} \gamma_{r^{+}}$, where $\gamma_{z} \in Z, \gamma_{i}$ is $\mathbb{G}$-good of depth $b_{i}$ with $b_{1}<\cdots<b_{k} \leq r$ and the sequence of centralizers $\mathbb{H}^{i}(\gamma)=C_{G}\left(\gamma_{z} \gamma_{1} \cdots \gamma_{i}\right)$ is strictly decreasing. In this case, we also write $\gamma_{\leq r}:=\gamma_{z} \cdot \gamma_{1} \cdots \gamma_{k}$

(2) Let $\gamma=\gamma_{z} \gamma_{1} \cdots \gamma_{k} \gamma_{r}$ be a good product as in (1). Define $\mathbb{H}^{\gamma, r}:=\mathbb{H}^{k}(\gamma)$. We will often write $\mathbb{H}^{\gamma}$ for $\mathbb{H}^{\gamma, r}$ for simplicity.

In [3], $\left(\gamma_{z}, \gamma_{1}, \cdots, \gamma_{k}\right)$ is called an $r^{+}$-normal approximation to $\gamma$. The following is similar to [3, Proposition 8.4].

Lemma 2.4.5. Suppose Hypothesis (C) holds. Suppose $\gamma=\gamma_{z} \gamma_{1} \cdots \gamma_{k}$ and $\gamma=$ $\gamma_{z}^{\prime} \gamma_{1}^{\prime} \cdots \gamma_{k^{\prime}}^{\prime}$ are two good products of $\gamma \bmod r^{+}$with $d\left(\gamma_{i}\right)=b_{i}$ and $d\left(\gamma_{i}^{\prime}\right)=b_{i}^{\prime}$. Write 
$\mathbb{H}^{i}=C_{\mathbb{G}}\left(\gamma_{z} \gamma_{1} \cdots \gamma_{i}\right)$ and $\mathbb{H}^{\prime i}=C_{\mathbb{G}}\left(\gamma_{z}^{\prime} \gamma_{1}^{\prime} \cdots \gamma_{i}^{\prime}\right)$. Then, we have $k=k^{\prime}, b_{i}=b_{i}^{\prime}$ and $\mathbb{H}^{i}=\mathbb{H}^{i}$.

Proof. We have $\gamma_{i}, \gamma_{j}^{\prime} \in C_{G}(\gamma) \subset\left(\cap_{i} H^{i}\right) \cap\left(\cap_{j} H^{\prime j}\right)=H^{k} \cap H^{\prime k^{\prime}}$ for $i \in\{z, 1, \cdots, k\}$ and $j \in\left\{z, 1, \cdots, k^{\prime}\right\}$.

Note that $d(\gamma)=b_{1}=b_{1}^{\prime}$. Since $\gamma_{z}^{\prime} \gamma_{1}^{\prime} \in \gamma_{z} \gamma_{1} H_{b_{1}^{+}}^{1}, \gamma_{z} \gamma_{1} \in \gamma_{z}^{\prime} \gamma_{1}^{\prime} H_{b_{1}^{+}}^{\prime 1}$ and $\gamma_{z} \gamma_{1}$ and $\gamma_{z}^{\prime} \gamma_{1}^{\prime}$ are $\mathbb{G}$-good mod center, $H^{1}=H^{\prime 1}$ by Lemma 2.4.1. By induction, suppose that $b_{j}=b_{j}^{\prime}$ and $\mathbb{H}^{j}=\mathbb{H}^{\prime j}$ for $1 \leq j \leq i$. Write $\gamma^{i}=\gamma_{1} \cdots \gamma_{i}$ and $\gamma^{i}=\gamma_{1}^{\prime} \cdots \gamma_{i}^{\prime}$. Suppose $b_{i+1}<b_{i+1}^{\prime}$. Then, $\left(\gamma^{i}\right)^{-1} \gamma^{i} \in \gamma_{i+1} H_{b_{i+1}^{+}}^{i+1}$. Since $\mathbb{G}$-good element $\gamma_{i+1}$ is also $\mathbb{H}^{i}$-good, we have $H^{i} \subset C_{G}\left(\left(\gamma^{i}\right)^{-1} \gamma^{i}\right) \subset H^{i+1}$ by Lemma 2.4.1(2). This is a contradiction to $H^{i} \subsetneq H^{i+1}$. Hence, $b_{i+1}=b_{i+1}^{\prime}$. Now we have

(i) $d\left(\gamma_{i+1}\right)=d\left(\gamma_{i+1}^{\prime} \gamma^{\prime i}\left(\gamma^{i}\right)^{-1}\right)=b_{i+1}$;

(ii) $\gamma^{\prime i}\left(\gamma^{i}\right)^{-1} \in Z\left(H^{i}\right)$;

(iii) $\gamma_{i+1}, \gamma_{i+1}^{\prime} \gamma^{\prime i}\left(\gamma^{i}\right)^{-1}$ are $\mathbb{H}^{i}$-good.

Combining (i)-(iii), it follows that $C_{\mathbb{G}}\left(\gamma^{i+1}\right)=C_{\mathbb{H}^{i}}\left(\gamma_{i+1}\right)=C_{\mathbb{H}^{i}}\left(\gamma_{i+1}^{\prime}\right)=C_{\mathbb{G}}\left(\gamma^{\prime i+1}\right)$. Hence, $\mathbb{H}^{i+1}=\mathbb{H}^{\prime i+1}$. Similarly, one can show $k=k^{\prime}$.

2.5. Decomposition of $G^{\text {rig }}$. We first observe that $G^{\text {rig }}=Z \cdot\left(\bigcup_{x \in \mathcal{B}(G)} \operatorname{Stab}_{G}(x)\right)$, and $Z G_{0} \subset G^{\text {rig }}$ where $G_{0}=\bigcup_{x \in \mathcal{B}(G)} G_{x, 0}$.

Definition 2.5.1. Let $\gamma, \gamma^{\prime} \in G^{\text {rig }}$.

(1) Suppose that $\gamma$ and $\gamma^{\prime}$ are $\mathbb{G}$-good mod center. We say that they are $\mathbb{G}$-good $r^{+}$-equivalent and write $\gamma \stackrel{g}{\sim} \gamma^{\prime}$ if there are $g \in G$ and a maximal torus $T$ such that ${ }^{g} \gamma^{\prime} \in \gamma T_{t^{+}} \subset T$ where $t=\min \{d(\gamma), r\}$. We write a $\mathbb{G}$-good $r^{+}$-equivalence class of $\gamma$ as $[\gamma]_{\mathrm{g}}$. Let

$[Z]_{r}^{\mathrm{g}}:=\left\{[z]_{\mathrm{g}} \mid \bar{z} \in Z / Z_{r^{+}}\right\}$,

$\mathcal{S}_{r}^{\mathrm{g}} \quad:=[Z]_{r}^{\mathrm{g}} \cup\left\{[\gamma]_{\mathrm{g}} \mid \gamma\right.$ is $\mathbb{G}$-good mod center of depth $\left.d(\gamma) \leq r\right\}$.

(2) For $\gamma, \gamma^{\prime} \in G^{\text {rig }}$, we say $\gamma$ and $\gamma^{\prime}$ are $r^{+}$-equivalent and write $\gamma \sim \gamma^{\prime}$ if there are $g \in G$, a maximal torus $T$, and good products of $\gamma$ and $\gamma^{\prime}$ so that ${ }^{g} \gamma_{\leq r}^{\prime} \in \gamma_{\leq r} T_{r^{+}} \subset T$.

We write an $r^{+}$-equivalence class of $\gamma$ as $[\gamma]$, and let $\mathcal{S}_{r}$ be the set of $r^{+}$-equivalence classes of semisimple compact-modulo-center elements.

Lemma 2.4.5 and the following proposition shows that the definition in (2) does not depend on the choice of truncation $\gamma_{\leq r}$ and $\gamma_{\leq r}^{\prime}$.

Proposition 2.5.2. Suppose Hypotheses (A)-(D) and 1-4 hold. Then, we have the following:

(1) For $\gamma, \gamma^{\prime} \in G^{\mathrm{rig}},{ }^{G}\left(\gamma H_{r^{+}}^{\gamma}\right) \cap{ }^{G}\left(\gamma^{\prime} H_{r^{+}}^{\gamma^{\prime}}\right) \neq \emptyset$ if and only if $\gamma$ and $\gamma^{\prime}$ are $r^{+}$-equivalent.

(2) If $\gamma \sim \gamma^{\prime},{ }^{G}\left(\gamma H_{r^{+}}^{\gamma}\right)={ }^{G}\left(\gamma^{\prime} H_{r^{+}}^{\gamma^{\prime}}\right)$.

(3)

$$
\bigsqcup_{[\gamma] \in \mathcal{S}_{r}}{ }^{G}\left(\gamma H_{r^{+}}^{\gamma}\right)=G^{\mathrm{rig}}
$$

(4) Each $^{G}\left(\gamma H_{r^{+}}^{\gamma}\right)$ is open and closed. 
Proof. (1) For $\Leftarrow$, by Lemma 2.4.5, we have $\gamma H_{r^{+}}^{\gamma}=\gamma_{\leq r} H_{r^{+}}^{\gamma}$ and $\gamma^{\prime} H_{r^{+}}^{\gamma^{\prime}}=\gamma_{\leq r}^{\prime} H_{r^{+}}^{\gamma^{\prime}}$ independent of the choices of $\gamma_{\leq r}$ and $\gamma_{\leq r}^{\prime}$. Since $\gamma_{\leq r} T_{r} \subset H^{\gamma}$, hence $\gamma_{\leq r}^{\prime} \in$ $G\left(\gamma H_{r^{+}}^{\gamma}\right) \cap{ }^{G}\left(\gamma^{\prime} H_{r^{+}}^{\gamma^{\prime}}\right)$.

For $\Rightarrow$ and (2), without loss of generality, one may assume that $\gamma H_{r^{+}}^{\gamma} \cap \gamma^{\prime} H_{r^{+}}^{\gamma^{\prime}} \neq \emptyset$. One may also assume that $\gamma=\gamma_{1} \cdots \gamma_{k}$ and $\gamma^{\prime}=\gamma_{1}^{\prime} \cdots \gamma_{k^{\prime}}^{\prime}$ with $b_{k}, b_{k^{\prime}}^{\prime} \leq r$, that is, $\gamma_{r^{+}}=\gamma_{r^{+}}^{\prime}=1$. Let $\delta \in \gamma H_{r^{+}}^{\gamma} \cap \gamma^{\prime} H_{r^{+}}^{\gamma^{\prime}}$. Then, $\delta=\gamma \cdot h=\gamma^{\prime} \cdot h^{\prime}$ with $h \in H_{r^{+}}^{\gamma}$ and $h^{\prime} \in H_{r^{+}}^{\gamma^{\prime}}$. Let $h=h_{s} h_{u}$ (resp., $h^{\prime}=h_{s}^{\prime} h_{u}^{\prime}$ ) be the Jordan decomposition of $h$ in $H^{\gamma}$ (resp., $h^{\prime}$ in $H^{\gamma^{\prime}}$ ). Then, $\gamma h_{s} h_{u}$ and $\gamma^{\prime} h_{s}^{\prime} h_{u}^{\prime}$ are two expressions of the Jordan decomposition of $\delta$. By the uniqueness of Jordan decomposition, $\gamma h_{s}=\gamma^{\prime} h_{s}^{\prime}$. Note $d\left(h_{s}\right)=d(h)>r$ and $d\left(h_{s}^{\prime}\right)=d\left(h^{\prime}\right)>r$. By applying Lemma 2.4.5 to $\gamma h_{s}$, we have $H^{\gamma h_{s}}=H^{\gamma}=H^{\gamma^{\prime}}$. Hence, $\gamma H_{r^{+}}^{\gamma}=\gamma h_{s} H_{r^{+}}^{\gamma}=\gamma^{\prime} H_{r^{+}}^{\gamma^{\prime}}$ and $\gamma \sim \gamma^{\prime}$.

For (3), write $G_{\mathcal{S}_{r}}$ for $\bigsqcup_{[\gamma] \in \mathcal{S}_{r}}{ }^{G}\left(\gamma H_{r^{+}}^{\gamma}\right)$. Clearly, $G_{\mathcal{S}_{r}} \subset G^{\text {rig }}$, and $G_{\mathcal{S}_{r}}$ is a disjoint union by (1). Conversely, for any $g \in G^{\text {rig }}$, there is $x \in \mathcal{B}(G)$ and $z \in Z$ so that $g z \in \operatorname{Stab}_{G}(x)$. By [26, Theorem 2.38], we have the topological Jordan decomposition of $g z=g_{\mathrm{a}} g_{0^{+}}$with $g_{\mathrm{a}}$ absolutely semisimple and $g_{0^{+}} \in G_{0^{+}}^{\prime}$ where $G^{\prime}=C_{G}\left(g_{\mathrm{a}}\right)$. By Lemma 2.3.4, $x \in \mathcal{B}\left(G^{\prime}\right)$. By Corollary 2.3.5, we have $g_{\mathrm{a}} \in$ $\operatorname{Stab}_{G}(x)$. Let $g_{0^{+}}=g_{s} g_{u}$ be the Jordan decomposition of $g_{0^{+}}$in $G^{\prime}$. Let $g_{s}=$ $g_{1} \cdots g_{k} g_{r}+$ be a good product of $g_{s} \bmod r^{+}$with $d\left(g_{i}\right)=b_{i}$. Then, since $g_{u}$ commutes with $g_{s}, g_{u} \in C_{G^{\prime}}\left(g_{1} \cdots g_{k}\right)=H^{g_{\mathrm{a}} g_{s}}$ by Lemma 2.4.1(2) and thus $g z=$ $g_{\mathrm{a}} g_{s} g_{u} \in g_{\mathrm{a}} g_{s} H_{r^{+}}^{g_{\mathrm{a}} g_{s}}$. Hence, $g \in G_{\mathcal{S}_{r}}$ and $G^{\mathrm{rig}} \subset G_{\mathcal{S}_{r}}$.

For (4), let $\gamma=\gamma_{z} \gamma_{1} \cdots \gamma_{k} \gamma_{r^{+}}$and let $H^{i}$ be as in Proposition 2.4.3. We may assume $\gamma_{r^{+}}=1$. Let $g \in G$ and $h \in H_{r^{+}}^{\gamma}$ so that ${ }^{g}(\gamma h) \in{ }^{G}\left(\gamma H_{r^{+}}^{\gamma}\right)$. We may assume that $g=1$. Let $y \in \mathcal{B}\left(H^{\gamma}\right)$ with $\gamma h \in \gamma H_{y, r^{+}}^{\gamma} \subset \gamma H_{r^{+}}^{\gamma}$. By Lemma 2.3.2. we have $H_{y,\left(r-b_{k}\right)^{+}}^{k-1}\left(\gamma_{k} H_{y, r^{+}}^{\gamma}\right)=\gamma_{k} H_{y, r^{+}}^{k-1}$ and thus $H_{y,\left(r-b_{k}\right)^{+}}^{k-1}\left(\gamma H_{y, r^{+}}^{\gamma}\right)=\gamma H_{y, r^{+}}^{k-1}$ since $\gamma_{1} \cdots \gamma_{k-1} \in Z\left(H^{k-1}\right)$. Inductively, setting $\mathbb{H}^{0}=\mathbb{G}$, we have ${ }_{y,\left(r-b_{i}\right)+}^{i-1}\left(\gamma H_{y, r^{+}}^{\gamma}\right)=$ $\gamma H_{y, r^{+}}^{i-1}$ for $i=1, \cdots, k$. Hence, $\gamma h \in \gamma G_{y, r^{+}} \subset{ }^{G}\left(\gamma H_{r^{+}}^{\gamma}\right)$ and hence ${ }^{G}\left(\gamma H_{r^{+}}^{\gamma}\right)$ is open. It is also closed since its complement is open.

Corollary 2.5.3. Suppose Hypotheses (A)-(D) and 1-4 hold.

(1) For $g \in G^{\text {rig }}$ with $d(g)<\infty$, we have $g=\gamma \cdot u$ for a $\mathbb{G}$-good mod center element $\gamma$ of depth $d(g)$ and $u \in G_{d(\gamma)^{+}}^{\gamma}$ where $\mathbb{G}^{\gamma}=C_{\mathbb{G}}(\gamma)$.

(2) We have

$$
G^{\mathrm{rig}}=\left(\bigsqcup_{[\gamma]_{\mathrm{g}} \in \mathcal{S}_{r}^{g} \backslash[Z]_{r}^{g}} G\left(\gamma G_{d(\gamma)^{+}}^{\gamma}\right)\right) \sqcup\left(\bigsqcup_{[z]_{\mathrm{g}} \in[Z]_{r}^{g}} z G_{r^{+}}\right) .
$$

Proof. (1) Applying the above proposition when $r:=d(g)$, we have $g \in \gamma G_{r^{+}}^{\gamma}$ for a $\mathbb{G}$-good mod center element $\gamma$ of depth $d(g)$.

(2) By (1) $G^{\mathrm{rig}}=\left(\bigcup_{[\gamma]_{\mathrm{g}} \in \mathcal{S}_{r}^{\mathrm{g}} \backslash[Z]_{r}^{\mathrm{g}}} G\left(\gamma G_{d(\gamma)^{+}}^{\gamma}\right)\right) \cup\left(\bigcup_{[z]_{\mathrm{g}} \in[Z]_{r}^{\mathrm{g}}} z G_{r^{+}}\right)$.

To prove the disjointness, suppose $[\gamma]_{\mathrm{g}},\left[\gamma^{\prime}\right]_{\mathrm{g}} \notin[Z]_{\mathrm{g}}$. If $G\left(\gamma G_{d(\gamma)^{+}}^{\gamma}\right) \cap^{G}\left(\gamma^{\prime} G_{d\left(\gamma^{\prime}\right)^{+}}^{\gamma^{\prime}}\right)$ $\neq \emptyset, d(\gamma)=d\left(\gamma^{\prime}\right)$ and ${ }^{G}\left(\gamma G_{d(\gamma)^{+}}^{\gamma}\right)={ }^{G}\left(\gamma^{\prime} G_{d\left(\gamma^{\prime}\right)^{+}}^{\gamma^{\prime}}\right)$ follows from the above lemma by setting $r=d(\gamma)$. The other cases are easier. 


\section{Descents}

3.1. Theorem A. From now on, we fix $r \in \mathbb{R}_{>0}$. For any $[\gamma] \in \mathcal{S}_{r}$, let $\mathcal{H}\left({ }^{G}\left(\gamma H_{r^{+}}^{\gamma}\right)\right)$ be the $\mathbb{Z}\left[\frac{1}{p}\right]$-submodule of $\mathcal{H}$ consisting of functions supported in ${ }^{G}\left(\gamma H_{r^{+}}^{\gamma}\right)$ and let $\overline{\mathcal{H}}\left({ }^{G}\left(\gamma H_{r^{+}}^{\gamma}\right)\right)$ be its image in $\overline{\mathcal{H}}$. By Lemma 1.2 .3 and Proposition 2.5.2, we have

$$
\mathcal{H}^{\text {rig }}=\bigoplus_{[\gamma] \in \mathcal{S}_{r}} \mathcal{H}\left({ }^{G}\left(\gamma H_{r^{+}}^{\gamma}\right)\right), \quad \overline{\mathcal{H}}^{\text {rig }}=\bigoplus_{[\gamma] \in \mathcal{S}_{r}} \overline{\mathcal{H}}\left({ }^{G}\left(\gamma H_{r^{+}}^{\gamma}\right)\right) .
$$

Let $\mathcal{H}_{r^{+}}\left({ }^{G}\left(\gamma H_{r^{+}}^{\gamma}\right)\right):=\mathcal{H}_{r^{+}} \cap \mathcal{H}\left({ }^{G}\left(\gamma H_{r^{+}}^{\gamma}\right)\right)$. Then we have

$$
\mathcal{H}_{r^{+}}^{\text {rig } \supset} \bigoplus_{[\gamma] \in \mathcal{S}_{r}} \mathcal{H}_{r^{+}}\left({ }^{G}\left(\gamma H_{r^{+}}^{\gamma}\right)\right) .
$$

Note that the intersection of a double coset of $G_{x, r^{+}}$with a given $G$-domain ${ }^{G}\left(\gamma H_{r^{+}}^{\gamma}\right)$, in general, is not closed under the left (or equivalently, right) multiplication of $G_{x, r^{+}}$. Thus we have

$$
\mathcal{H}_{r^{+}}^{\mathrm{rig}} \neq \bigoplus_{[\gamma] \in \mathcal{S}_{r}} \mathcal{H}_{r^{+}}\left({ }^{G}\left(\gamma H_{r^{+}}^{\gamma}\right)\right)
$$

Let $\overline{\mathcal{H}}_{r^{+}}\left({ }^{G}\left(\gamma H_{r^{+}}^{\gamma}\right)\right)$ be the image of $\mathcal{H}_{r^{+}}\left({ }^{G}\left(\gamma H_{r^{+}}^{\gamma}\right)\right)$ in $\overline{\mathcal{H}}_{r^{+}}$. In other words, $\overline{\mathcal{H}}_{r^{+}}\left({ }^{G}\left(\gamma H_{r^{+}}^{\gamma}\right)\right)$ is the $\mathbb{Z}\left[\frac{1}{p}\right]$-submodule of $\overline{\mathcal{H}}$ consisting of elements represented by functions in $\mathcal{H}_{r^{+}}\left({ }^{G}\left(\gamma H_{r^{+}}^{\gamma}\right)\right)$.

The main purpose of this section is to show that we still have the desired direct sum decomposition of $\overline{\mathcal{H}}_{r^{+}}^{\text {rig }}=\bigoplus_{[\gamma] \in \mathcal{S}_{r}} \overline{\mathcal{H}}_{r^{+}}\left({ }^{G}\left(\gamma H_{r^{+}}^{\gamma}\right)\right)$.

\section{Definition 3.1.1.}

(1) For any $s \in \widetilde{\mathbb{R}}_{\geq 0}$, we define

$$
\mathcal{H}_{s}^{G}=\sum_{x \in \mathcal{B}(G)} C_{c}\left(G / G_{x, s}\right) .
$$

For $s, t \in \widetilde{\mathbb{R}}$, with $0<t<s$, and $\gamma_{z} \in Z\left(\bmod Z_{r^{+}}\right)$, define

$$
\begin{gathered}
\mathcal{H}_{t, s}^{G}\left(\gamma_{z}\right)=\sum_{x \in \mathcal{B}(G)} C_{c}\left(\left(\gamma_{z} \cdot G_{x, t}\right) / G_{x, s}\right), \\
\mathcal{H}_{t, s}^{G, b}\left(\gamma_{z}\right)=\sum_{x \in \mathcal{B}(G)} C_{c}\left(\left(\gamma_{z} \cdot\left(G_{x, t} \cap G_{t^{+}}\right)\right) / G_{x, s}\right) .
\end{gathered}
$$

We note that $\mathcal{H}_{s, s^{+}}^{G, b}\left(\gamma_{z}\right)$ is spanned by $\mathbb{1}_{X}$, where

$$
X=\gamma_{z} g G_{x, s^{+}} \in \gamma_{z} G_{x, s} / G_{x, s^{+}}
$$

for some $g \in G_{x, s}$ and $x \in \mathcal{B}(G)$ with $g G_{x, s^{+}} \subset G_{s^{+}}$by [2, Corollary 3.7.8 and Corollary 3.7.10]. For simplicity, we will also write

$$
\mathcal{H}_{t, s}^{G}:=\mathcal{H}_{t, s}^{G}(1), \quad \mathcal{H}_{t, s}^{G, b}(1)=\mathcal{H}_{t, s}^{G, b} .
$$

(2) Let $\gamma=\gamma_{z} \gamma_{1} \cdots \gamma_{k} \gamma_{r^{+}} \in T$ be a good product of $\gamma$ with $d\left(\gamma_{i}\right)=b_{i}$.

(i) Let $\mathbb{H}^{\gamma}=C_{\mathbb{G}}\left(\gamma_{\leq r}\right)$. Define

$$
\mathcal{H}_{[\gamma]}^{G, b}:=\sum_{x \in \mathcal{B}\left(H^{\gamma}\right)} C_{c}\left(\left(\gamma_{\leq r} \cdot\left(H_{x, r}^{\gamma} \cap H_{r^{+}}^{\gamma}\right) G_{x, r^{+}}\right) / G_{x, r^{+}}\right) .
$$


In particular, for any $\gamma_{z} \in Z(G)$, we have

$$
\mathcal{H}_{\left[\gamma_{z}\right]}^{G, b}:=\sum_{x \in \mathcal{B}(G)} C_{c}\left(\left(\gamma_{z} \cdot\left(G_{x, r} \cap G_{r^{+}}\right) G_{x, r^{+}}\right) / G_{x, r^{+}}\right) .
$$

(ii) If $[\gamma]_{\mathrm{g}}:=\left[\gamma_{z} \gamma_{1}\right]_{\mathrm{g}} \in \mathcal{S}_{r}^{\mathrm{g}}$ with $\gamma_{1} \mathbb{G}$-good of depth $b_{1} \leq r$, define

$$
\mathcal{H}_{[\gamma]_{\mathrm{g}}}^{G, b}:=\sum_{x \in \mathcal{B}\left(H^{1}\right)} C_{c}\left(\left(\gamma_{z} \gamma_{1} \cdot\left(H_{x, b_{1}}^{1} \cap H_{b_{1}^{+}}^{1}\right) G_{x, r^{+}}\right) / G_{x, r^{+}}\right),
$$

where $\mathbb{H}^{1}=C_{\mathbb{G}}\left(\gamma_{1}\right)$. If $[\gamma]_{\mathrm{g}}=\left[\gamma_{z}\right]_{\mathrm{g}}$, let

$$
\mathcal{H}_{\left[\gamma_{z}\right]_{\mathrm{g}}}^{G, b}:=\mathcal{H}_{\left[\gamma_{z}\right]}^{G, b} \text {. }
$$

Note that we have $\mathcal{H}_{[1]_{\mathrm{g}}}^{G, b}=\mathcal{H}_{[1]}^{G, b}=\mathcal{H}_{r, r^{+}}^{G, b}(1)$.

In all cases, we denote the image of each $\mathbb{Z}\left[\frac{1}{p}\right]$-submodule in the cocenter $\overline{\mathcal{H}}$ using - e.g., $\overline{\mathcal{H}}_{s}^{G}, \overline{\mathcal{H}}_{t, s, b}^{G, \text { }}$, etc.

Theorem 3.1.2. Suppose Hypotheses (A)-(D) and 1-4 hold.

(1) $\overline{\mathcal{H}}_{r^{+}}^{\text {rig }}=\bigoplus_{[\gamma] \in \mathcal{S}_{r}} \overline{\mathcal{H}}_{r^{+}}\left({ }^{G}\left(\gamma H_{r^{+}}^{\gamma}\right)\right)$.

(2) For any $[\gamma] \in \mathcal{S}_{r}, \overline{\mathcal{H}}_{r+}\left({ }^{G}\left(\gamma H_{r^{+}}^{\gamma}\right)\right)=\overline{\mathcal{H}}_{[\gamma]}^{G, b}$.

We will prove the above theorem in the rest of this section. We first need some lemmas.

3.2. Some lemmas. The following is [9, Lemma 4.5.1].

Lemma 3.2.1. Suppose Hypotheses (DB) and 2 hold. Let $x \in \mathcal{B}(\mathbb{G}, F)$ and suppose $s<r$. Let $\mathbb{S} \subset \mathbb{G}$ be a maximal $k$-split torus of $\mathbb{G}$ such that $x \in \mathcal{A}(\mathbb{S}, F)$. If $u \in\left(\mathcal{U} G_{x, s^{+}} \cap\left(G_{x, s} \backslash G_{x, s^{+}}\right)\right)$, then there exist $v \in G_{x}\left(u G_{x, s^{+}}\right)$and $\lambda \in \mathbf{X}_{*}(\mathbb{S}, F)$ such that for sufficiently small $\epsilon>0$, we have

(1) $v G_{x, s^{+}} \subset G_{x+\epsilon \lambda, s^{+}}$and

(2) $v v^{\prime} G_{x+\epsilon \lambda, r^{+}} \subset G_{x,(r-s)}\left(v v^{\prime} G_{x, r^{+}}\right)$for any $v^{\prime} \in G_{x, s^{+}}$.

Definition 3.2.2 (4]). For any $g \in G$, the displacement function $\mathrm{d}_{g}: \mathcal{B}(G) \rightarrow \mathbb{R}$ is defined as $\mathrm{d}_{g}(x)=\operatorname{dist}(\bar{x}, g \bar{x})$ where $\operatorname{dist}(\bar{x}, g \bar{x})$ is a geodesic distance in the reduced building $\mathcal{B}(\bar{G})$ between $\bar{x}$ and $g \bar{x}$ where $\bar{x}$ is the image of $x$ in $\mathcal{B}(\bar{G})$. Define $\mathrm{d}(g):=\min \left\{\mathrm{d}_{g}(x) \mid x \in \mathcal{B}(G)\right\}$. For any subset $S \subset \mathcal{B}(G)$ with compact image in $\mathcal{B}(\bar{G})$, define $\mathrm{d}_{S}(g):=\min \left\{\mathrm{d}_{g}(x) \mid x \in S\right\}$. Note that $\mathrm{d}_{S}$ is well defined since $S$ has a compact image in $\mathcal{B}(\bar{G})$.

We would also need the notion of generalized $r$-facets. In [8], they are defined as certain subsets of the reduced building $\mathcal{B}(\bar{G})$. One can define generalized $r$-facets on the extended building $\mathcal{B}(G)$ in a similar way.

Definition 3.2.3 ([8]). For $x \in \mathcal{B}(G)$, define

$$
\begin{aligned}
F^{*}(x): & =\left\{y \in \mathcal{B}(G) \mid \mathfrak{g}_{x, r}=\mathfrak{g}_{y, r} \text { and } \mathfrak{g}_{x, r^{+}}=\mathfrak{g}_{y, r^{+}}\right\} \\
& =\left\{y \in \mathcal{B}(G) \mid G_{x, r}=G_{y, r} \text { and } G_{x, r^{+}}=G_{y, r^{+}}\right\}, \\
\mathcal{F}(r): & =\left\{F^{*}(x) \mid x \in \mathcal{B}(G)\right\} .
\end{aligned}
$$


An element in $\mathcal{F}(r)$ is called a generalized $r$-facet in $\mathcal{B}(G)$. We will often write $F^{*}$ for $F^{*}(x)$ when there is no confusion. Note that the closure $\bar{F}^{*}$ of $F^{*} \in F^{*}(r)$ has a compact image in $\mathcal{B}(\bar{G})$. For $F^{*}=F^{*}(x) \in \mathcal{F}(r)$, define

$$
\begin{aligned}
\mathfrak{g}_{F^{*}}:=\mathfrak{g}_{x, r}, & \mathfrak{g}_{F^{*}}^{+}:=\mathfrak{g}_{x, r^{+}}, \\
G_{F^{*}}:=G_{x, r}, & G_{F^{*}}^{+}:=G_{x, r^{+}} .
\end{aligned}
$$

Remarks 3.2.4. Let $y \in \mathcal{B}(G)$ and let $\mathbb{S}$ be a maximal $F$-split torus of $\mathbb{G}$ with $y \in \mathcal{A}(S)$. Let $C$ be an alcove (0-facet of maximal dimension) with $y \in \bar{C} \subset \mathcal{A}(S)$. For $g \in G$, there are $n \in N_{G}(S)$ and $b_{i} \in G_{C}$ with $g=b_{1} n b_{2}$ where $G_{C}=G_{x, 0}$ for $x \in C$. Define $g^{\prime}=b_{1}^{-1} g=n b_{2} b_{1}$. Then, we have the following:

(1) For $r \geq 0$, since $b_{1} \in N_{G}\left(G_{y, r}\right) \cap N_{G}\left(G_{y, r^{+}}\right)$, we have $b_{1} \in \operatorname{Stab}_{G}\left(F^{*}\right)$ where $F^{*}$ is the $r$-facet containing $y$.

(2) We have $\mathbb{1}_{g G_{F^{*}}^{+}} \equiv \mathbb{1}_{g^{\prime} G_{F^{*}}^{+}} \bmod [\mathcal{H}, \mathcal{H}]$. Here for $X \subset G, \mathbb{1}_{X}$ denotes the characteristic function with support $X$.

(3) Since $y \in \bar{C}$, we have $g^{\prime} y=n b_{2} b_{1} y=n y \in \mathcal{A}(S)$.

(4) $\mathrm{d}_{g^{\prime}}(y)=\mathrm{d}_{g}(y)$.

The proof of the following lemma is adapted from that of [9, Corollary 4.2.9]. We include the proof for completeness.

Lemma 3.2.5. Let $F^{*} \in \mathcal{F}(r)$ and $g \in G$. Suppose $\mathrm{d}(g)=0$ and $m:=\mathrm{d}_{\bar{F}^{*}}(g)>0$. Then, there is a finite set $\left\{g_{i}\right\}$ and constants $c_{i} \in \mathbb{Z}\left[\frac{1}{p}\right]$ and $F_{i}^{*} \in \mathcal{F}(r)$ such that

(1) $\mathrm{d}_{\bar{F}^{*}}(g)>\mathrm{d}_{\bar{F}_{i}^{*}}\left(g_{i}\right)$ for each $i$, and

(2) $\mathbb{1}_{g G_{F^{*}}^{+}} \equiv \sum_{i} c_{i} \mathbb{1}_{g_{i} G_{F_{i}^{*}}^{+}} \bmod [\mathcal{H}, \mathcal{H}]$

Proof. We divide the proof into two cases.

Case 1 . There is $y \in F^{*}$ with $\mathrm{d}_{g}(y)=\mathrm{d}_{\bar{F}^{*}}(g)$.

Choose $\mathbb{S}$ and $C$ as in Remarks 3.2.4, and keep the notation from there. Write $[y, n y]$ for the geodesic in $\mathcal{A}(S)$ between $y$ and $n y$. Observe that $[y, n y] \cap \bar{F}^{*}=\{y\}$ (see the proof of [9, Lemma 4.2.6] for details). Let $F_{1}^{*} \in \mathcal{F}(r)$ be the first generalized $r$-facet that $\left(y, g^{\prime} y\right]=(y, n y]$ passes through when traveling from $y$ to $n y$. Note that $\bar{F}_{1}^{*} \cap \mathcal{A} \neq \emptyset$. Since $F^{*} \subset \bar{F}_{1}^{*}$ thus $F^{*} \cap \mathcal{A} \subset \bar{F}_{1}^{*} \cap \mathcal{A}$. Note that $G_{F^{*}}^{+} \subset G_{\bar{F}_{1}^{*}}^{+}$. Let

$$
Q:=\left\{\psi \in \Psi(\mathbb{S}, \mathbb{G}, F)|\psi|\left(F_{1}^{*} \cap \mathcal{A}\right)>r \text { and } \psi \mid\left(F^{*} \cap \mathcal{A}\right)=r\right\}
$$

Then,

$$
G_{F_{1}^{*}}^{+}=G_{F^{*}}^{+} \cdot \prod_{\psi \in Q} U_{\psi}
$$

where the product over $Q$ may be taken in any order. Fix $\psi \in Q$. Since $\left(n^{-1} \psi\right)(y)=$ $\psi(n y)>r$, we have ${ }^{n^{-1}} U_{\psi}=U_{n^{-1} \psi} \subset G_{F^{*}}^{+}$. We also have $U_{\psi}^{+} \subset G_{F^{*}}^{+}$. By (2) of 
the above remarks, we have

$$
\begin{aligned}
\mathbb{1}_{g \cdot G_{F^{*}}^{+}} & \equiv \mathbb{1}_{g^{\prime} \cdot G_{F^{*}}^{+}} \sum_{\bar{h} \in\left(\prod_{\psi \in Q} U_{\psi}\right) /\left(\prod_{\psi \in Q} U_{\psi}^{+}\right)} \mathbb{1}_{h^{-1} n b_{2} b_{1} G_{F^{*}}^{+} h} \sum_{\bar{h} \in\left(\prod_{\psi \in Q}\right.} \sum_{\left.U_{\psi}\right) /\left(\prod_{\psi \in Q} U_{\psi}^{+}\right)} \mathbb{1}_{g^{\prime} G_{F^{*}}^{+} h} \\
& \equiv c \cdot \mathbb{1}_{g^{\prime} G_{F_{1}^{*}}^{+}} \bmod [\mathcal{H}, \mathcal{H}],
\end{aligned}
$$

where the constant $c=\sharp\left(\left(\prod_{\psi \in Q} U_{\psi}\right) /\left(\prod_{\psi \in Q} U_{\psi}^{+}\right)\right)^{-1} \in \mathbb{Z}\left[\frac{1}{p}\right]$. Note that for all $z \in$ $F_{1}^{*} \cap\left(y, g^{\prime} y\right) \neq \emptyset$, we have from [9, Lemma 4.2.1] that $\mathrm{d}_{g^{\prime}}(z)<\mathrm{d}_{g^{\prime}}(y)$. Combining with (4) of the above remarks, we have

$$
\min _{x \in \bar{F}_{1}^{*}} \mathrm{~d}_{g^{\prime}}(x)<\mathrm{d}_{g^{\prime}}(y)=\mathrm{d}_{g}(y)=\min _{x \in \overline{F^{*}}} \mathrm{~d}_{g}(x) .
$$

Case 2. For all $x \in F^{*}, \mathrm{~d}_{g}(x)>\mathrm{d}_{\bar{F}^{*}}(g)$.

Choose $y \in \bar{F}^{*} \backslash F^{*}$ such that $\mathrm{d}_{\bar{F}^{*}}(g)=\mathrm{d}_{g}(y)$. There exists $\bar{F}_{1}^{*} \in \mathcal{F}(r)$ such that $y \in F_{1}^{*}$ and $F_{1}^{*} \subset \bar{F}^{*}$. Then,

$$
\mathbb{1}_{g G_{F^{*}}^{+}}=\sum_{\alpha \in G_{F^{*}}^{+} / G_{F_{1}^{*}}^{+}} \mathbb{1}_{g \alpha G_{F_{1}^{*}}^{+}} .
$$

Note that for all $\alpha \in G_{F^{*}}^{+}$, we have $\mathrm{d}_{g}(x)=\mathrm{d}_{g \alpha}(x)$ for all $x \in \bar{F}^{*}$ and $\mathrm{d}_{g \alpha}(y)=$ $\mathrm{d}_{\bar{F}_{1}^{*}}(g \alpha)$ for all $\alpha \in G_{F^{*}}^{+}$. Now, one can apply Case 1 to each summand $\mathbb{1}_{g \alpha G_{F_{1}^{*}}^{+}}$, $\alpha \in G_{F^{*}}^{+} / G_{F_{1}^{*}}^{+}$, and $\bar{F}_{1}^{*}$.

\subsection{Descents.}

Proposition 3.3.1. Let $g G_{y, r} \subset G^{\mathrm{rig}}$. Write $s:=d(y, g)$ and $t:=d(g)$. There exists a finite indexing set $\{i\},\left\{g_{i}\right\} \subset G$, and $c_{i} \in \mathbb{Z}\left[\frac{1}{p}\right]$ such that $\mathbb{1}_{g G_{y, r^{+}}} \equiv$ $\sum_{i} c_{i} \mathbb{1}_{g_{i} G_{y_{i}, r^{+}}}(\bmod [\mathcal{H}, \mathcal{H}])$ with $d\left(g_{i}\right)=d\left(g_{i}, y_{i}\right) \leq r$ or $g_{i} \in Z \cdot\left(G_{r^{+}} \cap G_{y_{i}, r}\right)$.

Note that $s \leq t$. Note also that if $s<r$, we have $d(y, g)=d\left(y, g^{\prime}\right)$ for all $g^{\prime} \in g G_{y, r^{+}}$.

Proof. We prove the statement in three cases below. Without loss of generality, we may assume that $g$ is compact.

Case 1. $s=t=0$ or $s>r$.

Done since $\mathbb{1}_{g G_{y, r}+}$ already satisfies the required condition. In particular, when $s>r, g G_{y, r^{+}}=z G_{y, r^{+}}$for some $z \in Z$.

Case 2. $d(g)=0$ and $g \in Z \operatorname{Stab}_{G}(x) \backslash Z \operatorname{Stab}_{G}(y)$.

In this case, $\mathrm{d}_{g}(y)>0$. Let $F^{*} \in \mathcal{F}(r)$ with $y \in \bar{F}^{*}$.

If $\mathrm{d}_{\bar{F}^{*}}(g)=0$, from Case 1 in Lemma 3.2.5. we may assume that there is $z \in \bar{F}^{*}$ such that $\mathrm{d}_{g}(z)=0$. Then, $g G_{y, r^{+}}=g G_{z, r^{+}} \subset \operatorname{Stab}_{G}(z)$, which reduces to Case 3 below. 
Now, let $\mathrm{d}_{\bar{F}^{*}}(g)>0$. By applying Lemma 3.2.5 repeatedly, we can write

$$
\mathbb{1}_{g G_{y, r}+}=\mathbb{1}_{g G_{F^{*}}^{+}}=\sum_{i} c_{i} \mathbb{1}_{g_{i} G_{F_{i}^{*}}^{+}}
$$

with $\{i\}$ a finite set, $F_{i}^{*} \in \mathcal{F}(r)$, and $\mathrm{d}_{F_{i}^{*}}\left(g_{i}\right)=0$ for all $i$. More precisely, applying Lemma 3.2 .5 repeatedly, we find a sequence of triples $\left(g_{j}, F_{j}^{*}, y_{j}\right) \in G \times \mathcal{F}(r) \times \mathcal{B}(G)$, $j \in \mathbb{N}$ such that $y_{j} \in F_{j}^{*}$ and

$$
\mathrm{d}_{g_{j}}\left(y_{j}\right)=\mathrm{d}_{\overline{F_{j}^{*}}}\left(g_{j}\right)>\mathrm{d}_{\overline{F_{j+1}^{*}}}\left(g_{j+1}\right)=\mathrm{d}_{g_{j+1}}\left(y_{j+1}\right)>0 .
$$

Since $\mathrm{d}_{g_{j}}\left(y_{j}\right)$ is a discrete decreasing sequence in $\mathbb{R}_{\geq 0}$, for sufficiently large $j$, $\mathrm{d}_{g_{j}}\left(y_{j}\right)=0$ (see the proof of [9, Theorem 4.1.4-(1)]).

Case $3.0 \leq s \leq t$.

If $t=s$, it is done.

Now, suppose that $t>s$. Then, $g \in G_{y, s} \cap \mathcal{U}_{G_{y, s^{+}}}$. We claim that there is a finite set $\{i\}$ such that

$$
\mathbb{1}_{g G_{y, r}+} \equiv \sum c_{i} \mathbb{1}_{g_{i} G_{x_{i}, r+}} \bmod [\mathcal{H}, \mathcal{H}]
$$

with $c_{i} \in \mathbb{Z}\left[\frac{1}{p}\right]$ and $d\left(x_{i}, g_{i}\right)>s$.

Let $F^{*} \subset \mathcal{B}(G)$ be the generalized $r$-facet with $y \in F^{*}$. Let $\mathbb{S}$ be a maximal, maximally $F$-split torus in $\mathbb{G}$ so that $F^{*} \subset \mathcal{A}(\mathbb{S}, F)$. Since $d(, g)$ is continuous on $\mathcal{B}(G)$, for fixed $g \in G, d(, g)$ restricted to $\bar{F}^{*}$ attains its maximum since the image of $\bar{F}^{*}$ in the reduced building is compact. That is, there is $s^{\prime} \in \mathbb{R}_{\geq 0}$ with $s^{\prime} \geq s$ and $x \in \bar{F}^{*}$ such that

$$
s^{\prime}=d(x, g) \geq d(w, g)
$$

for all $w \in \bar{F}^{*}$.

Since $x \in \bar{F}^{*}$, we have $\mathfrak{g}_{x, r^{+}} \subset \mathfrak{g}_{F^{*}}^{+} \subset \mathfrak{g}_{F^{*}} \subset \mathfrak{g}_{x, r}$. Therefore,

$$
\mathbb{1}_{g G_{y, r}+}=\sum_{\bar{\alpha} \in G_{y, r} / G_{x, r+}} \mathbb{1}_{g \cdot \alpha G_{x, r^{+}}} .
$$

Note that each $\alpha \in G_{x, r}$ and $d(x, g)=d(x, g \alpha)=s$.

Now, for each $g \alpha$, we will show that there is a $z_{\alpha} \in \mathcal{B}(G)$ such that $\mathbb{1}_{g \alpha G_{x, r}+}$ is a linear combination of characteristic functions of the form $\mathbb{1}_{g_{\alpha} G_{z_{\alpha}, r}+}$ with $g_{\alpha} \in$ $G_{z_{\alpha}, s^{+}}$(see (2) below).

Since we are treating each $g \alpha$, for simplicity of notation, we may write $u$ for $g \alpha$. Note that $u \in G_{x, s^{\prime}} \cap G_{s^{\prime+}} \subset \mathcal{U} G_{x, s^{\prime+}}$. Then, we can find $v \in G_{x}\left(u G_{x, s^{\prime}}\right)$ and $\lambda$ as in Lemma 3.2.1 so that for sufficiently small $\epsilon>0$, we have (i) $v G_{x, s^{\prime}} \subset G_{x+\epsilon \lambda, s^{\prime}}$ and (ii) $v v^{\prime} G_{x+\epsilon \lambda, r^{+}} \subset G_{x,(r-s)}\left(v v^{\prime} G_{x, r^{+}}\right)$for any $v^{\prime} \in G_{x, s^{\prime}}$. Fix $\epsilon$ satisfying (i) and (ii). Write $z=x+\epsilon \lambda$. Let

$$
\mathfrak{a}:=\left\{v^{-1} h v h^{-1} \mid \bar{h} \in G_{x,\left(r-s^{\prime}\right)} / G_{x,\left(r-s^{\prime}\right)^{+}}\right\} G_{x, r^{+}} .
$$

We have $\mathfrak{a} \subset G_{x, r} \subset G_{x, s^{\prime}}$ and $G_{z, r^{+}} \subset \mathfrak{a}$. Then,

$$
\begin{aligned}
\mathbb{1}_{u G_{x, r^{+}}} & \equiv \mathbb{1}_{v G_{x, r}+} \equiv c \cdot \sum_{\bar{h} \in G_{x,\left(r-s^{\prime}\right)} / G_{x,\left(r-s^{\prime}\right)^{+}}} \mathbb{1}_{h v h^{-1} G_{x, r}+} \bmod [\mathcal{H}, \mathcal{H}], \\
& \equiv c \cdot \mathbb{1}_{v \cdot \mathfrak{a}} \equiv c \cdot \sum_{\bar{\beta} \in \mathfrak{a} / G_{z, r^{+}}} \mathbb{1}_{v \beta G_{z, r^{+}}} \bmod
\end{aligned}
$$


where $c=\left(\sharp\left(G_{x,\left(r-s^{\prime}\right)} / G_{x,\left(r-s^{\prime}\right)+}\right)\right)^{-1} \in \mathbb{Z}\left[\frac{1}{p}\right]$. For all $\beta \in \mathfrak{a}$, we have $\mathbb{1}_{v \beta G_{z, r}+} \in$ $C\left(G_{z, s^{\prime}} / G_{z, r^{+}}\right)$, and the claim is now proved.

Now one can repeat the process for $\mathbb{1}_{g_{i} G_{x_{i}, r}+}$ in (1) until each coset satisfies $d\left(g_{i}\right)=d\left(x_{i}, g_{i}\right)$ or $d\left(x_{i}, g_{i}\right) \geq r$. This is a finite process as in the proof of [9, Theorem 4.1.4-(1)]. We omit the details.

Remarks 3.3.2. Suppose Hypotheses (A)-(D) and 1-4 hold. We observe the following: Suppose $g G_{y, t^{+}}$satisfies $t:=d(g)=d(y, g)$. Write $g=\gamma \cdot u$ such that $\gamma$ is a $\mathbb{G}$-good mod center element and $u \in G_{d(\gamma)}^{\prime}$ where $G^{\prime}=C_{G}(\gamma)$ (see Corollary 2.5.3). Then,

(1) $d(g)=d(\gamma)=d(y, g)=d(y, \gamma)$,

(2) $y \in \mathcal{B}\left(\mathbb{G}^{\prime}, F\right)$,

(3) $\gamma \in Z G_{y, t}^{\prime}, \quad u \in G_{y, t}^{\prime} \cap G_{t^{+}}^{\prime}$,

(4) $\mathbb{1}_{g G_{y, r^{+}}} \in C_{c}\left(\left(\gamma \cdot\left(G_{y, t}^{\prime} \cap G_{t^{+}}^{\prime}\right) G_{y, r^{+}}\right) / G_{y, r^{+}}\right)$.

The first equality $d(g)=d(\gamma)$ follows from Lemma 2.3.4 (2) is Lemma 2.3.1 and (3) is Corollary 2.3.5.

The following is a corollary of Proposition 3.3 .1

Corollary 3.3.3. Suppose Hypotheses (A)-(D), (DB), and 1-4 hold. Then, we have

$$
\overline{\mathcal{H}}_{r^{+}}^{\mathrm{rig}}=\bigoplus_{[\gamma]_{\mathrm{g}} \in \mathcal{S}_{r}^{\mathrm{g}}} \overline{\mathcal{H}}_{[\gamma]_{\mathrm{g}}}^{G, \mathrm{~b}} .
$$

Proof. It follows from Corollary 2.5.3, Proposition 3.3.1, Lemma 1.2.3, and Remarks 3.3.2(4).

\subsection{Descent via induction.}

Proposition 3.4.1. Let $\gamma=\gamma_{z} \gamma_{1}$ be a $\mathbb{G}$-good mod center element of depth $t \geq 0$, where $\gamma_{z} \in Z(G)$ and $\gamma_{1}$ is $\mathbb{G}$-good of depth $t$. Let $s \in \tilde{\mathbb{R}}$ with $s>t$. We set $G^{\prime}=$ $C_{G}(\gamma)$. Then the map $\mathbb{1}_{h G_{x, s}^{\prime}} \mapsto \frac{\mu_{G^{\prime}}\left(G_{x, s}^{\prime}\right)}{\mu_{G}\left(G_{x, s}\right)} \mathbb{1}_{\gamma h G_{x, s}}$ for $x \in \mathcal{B}\left(G^{\prime}\right)$ and $h \in G_{x, t}^{\prime} \cap G_{t^{+}}^{\prime}$ induces a well-defined map

$$
\bar{i}_{\gamma, s}: \overline{\mathcal{H}}_{t, s}^{G^{\prime}, b} \rightarrow \overline{\mathcal{H}}_{t, s}^{G}\left(\gamma_{z}\right) .
$$

Moreover, for any $s^{\prime} \in \tilde{\mathbb{R}}$ with $s^{\prime} \geq s$, we have the following commutative diagram:

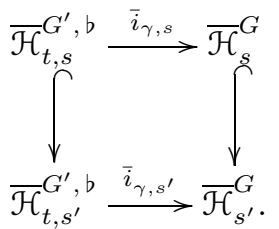

Remark 3.4.2. Note that the elements $\mathbb{1}_{h G_{x, s}^{\prime}}$ for $x \in \mathcal{B}(\gamma)$ and $h \in G_{x, t}^{\prime} \cap G_{t^{+}}^{\prime}$ are not linearly independent in $\mathcal{H}$. Thus the map $\mathbb{1}_{h G_{x, s}^{\prime}} \mapsto \frac{\mu_{G^{\prime}}\left(G_{x, s}^{\prime}\right)}{\mu_{G}\left(G_{x, s}\right)} \mathbb{1}_{\gamma h G_{x, s}}$ may not give a well-defined map from $\mathcal{H}_{t, s}^{G^{\prime}, b}$ to $\mathcal{H}_{s}^{G}$. However, we will see that it induces a well-defined map on the cocenter. 
Proof of Proposition 3.4.1. Let $x \in \mathcal{B}(\gamma)$ and $h \in G_{x, t}^{\prime} \cap G_{t^{+}}^{\prime}$. Let $\epsilon=s-t$. By Lemma 2.3.2, for any $k \in G_{x, s}$, there exists $g \in G_{x, \epsilon}$ such that ${ }^{g}(\gamma h k) \in$ $\gamma h G_{x, s}^{\prime}$. Note that $\left(G_{x, \epsilon}, G_{x, s}^{\prime}\right) \subset G_{x, s+\epsilon}$. Then $G_{x, s}^{\prime} G_{x, s+\epsilon}$ is a subgroup of $G$ and $g\left(G_{x, s}^{\prime} G_{x, s+\epsilon}\right)=G_{x, s}^{\prime} G_{x, s+\epsilon}$. We have ${ }^{g}\left(\gamma h k G_{x, s}^{\prime} G_{x, s+\epsilon}\right)=\gamma h G_{x, s}^{\prime} G_{x, s+\epsilon}$ and

$$
\mathbb{1}_{\gamma h k G_{x, s}^{\prime} G_{x, s+\epsilon}} \equiv \mathbb{1}_{\gamma h G_{x, s}^{\prime} G_{x, s+\epsilon}} \bmod [\mathcal{H}, \mathcal{H}] \text {. }
$$

Similarly, for any $n \in \mathbb{N}$ and $k \in G_{x, s+n \epsilon}$, we may write $G_{x, s}^{\prime}=\bigsqcup_{l} h_{l} G_{x, s+n \epsilon}^{\prime}$ for $h_{l} \in G_{x, s}^{\prime} / G_{x, s+n \epsilon}^{\prime}$. Since $G_{x, s+n \epsilon}^{\prime} \subset G_{x, s+n \epsilon}$, we have $h k G_{x, s}^{\prime}=\bigsqcup_{l} h h_{l} k_{l} G_{x, s+n \epsilon}^{\prime}$ for $k_{l}=h_{l}^{-1} k h_{l} \in G_{x, s+n \epsilon}$. Note that $h h_{l} \in G_{x, t}^{\prime} \cap G_{t^{+}}^{\prime}$. By Lemma 2.3.2, there exists $g_{l} \in G_{x,(n+1) \epsilon}$ such that ${ }^{g_{l}}\left(\gamma h h_{l} k_{l}\right) \in \gamma h h_{l} G_{x, s+n \epsilon}^{\prime}$. Note that $\left(G_{x,(n+1) \epsilon}, G_{x, s+n \epsilon}^{\prime}\right) \subset$ $G_{x, s+(2 n+1) \epsilon}$. Then $G_{x, s+n \epsilon}^{\prime} G_{x, s+(2 n+1) \epsilon}$ is a subgroup of $G$ and

$$
{ }^{g_{l}}\left(G_{x, s+n \epsilon}^{\prime} G_{x, s+2(n+1) \epsilon}\right)=G_{x, s+n \epsilon}^{\prime} G_{x, s+(2 n+1) \epsilon} .
$$

We have ${ }^{g_{l}}\left(\gamma h h_{l} k_{l} G_{x, s+n \epsilon}^{\prime} G_{x, s+(2 n+1) \epsilon}\right)=\gamma h h_{l} G_{x, s+n \epsilon}^{\prime} G_{x, s+(2 n+1) \epsilon}$ and

$$
\begin{aligned}
& \mathbb{1}_{\gamma h k G_{x, s}^{\prime} G_{x, s+(2 n+1) \epsilon}}=\sum_{l} \mathbb{1}_{\gamma h_{l} k_{l} G_{x, s+n \epsilon}^{\prime} G_{x, s+(2 n+1) \epsilon}} \\
& \equiv \sum_{l} \mathbb{1}_{\gamma h h_{l} G_{x, s+n \epsilon}^{\prime}} G_{x, s+(2 n+1) \epsilon} \\
& \equiv \mathbb{1}_{\gamma h G_{x, s}^{\prime} G_{x, s+(2 n+1) \epsilon}} \bmod [\mathcal{H}, \mathcal{H}] \text {. }
\end{aligned}
$$

In particular, we have

$$
\begin{aligned}
\mathbb{1}_{\gamma h G_{x, s}} & \equiv \frac{\mu_{G}\left(G_{x, s}\right)}{\mu_{G}\left(G_{x, s}^{\prime} G_{x, s+\epsilon}\right)} \mathbb{1}_{\gamma h G_{x, s}^{\prime} G_{x, s+\epsilon}} \\
& \equiv \cdots \\
& \equiv \frac{\mu_{G}\left(G_{x, s}\right)}{\mu_{G}\left(G_{x, s}^{\prime} G_{x, s+(2 n+1) \epsilon}\right)} \mathbb{1}_{\gamma h G_{x, s}^{\prime} G_{x, s+(2 n+1) \epsilon}} \bmod [\mathcal{H}, \mathcal{H}] .
\end{aligned}
$$

Moreover, for any open compact subgroup $K$ with $G_{x, s+(2 n+1) \epsilon} \subset K \subset G_{x, s+n \epsilon}$, we have $\mathbb{1}_{\gamma h G_{x, s}^{\prime} K} \equiv \frac{\mu_{G}\left(G_{x, s}^{\prime} K\right)}{\mu_{G}\left(G_{x, s}^{\prime} G_{x, s+(2 n+1) \epsilon}\right)} \mathbb{1}_{\gamma h G_{x, s}^{\prime} G_{x, s+(2 n+1) \epsilon}} \bmod [\mathcal{H}, \mathcal{H}]$ and hence

$$
\mathbb{1}_{\gamma h G_{x, s}} \equiv \frac{\mu_{G}\left(G_{x, s}\right)}{\mu_{G}\left(G_{x, s}^{\prime} K\right)} \mathbb{1}_{\gamma h G_{x, s}^{\prime} K} \quad \bmod [\mathcal{H}, \mathcal{H}]
$$

Now suppose that $\sum_{i} a_{i} \mathbb{1}_{h_{i} G_{x_{i}, s}^{\prime}}=0 \in \mathcal{H}_{t, s}^{G^{\prime}, b}$. We may choose a sufficiently large $n \in \mathbb{N}$ such that the subgroup generated by $G_{x_{i}, s+(2 n+1) \epsilon}$ for all $i$ is contained in $K:=\cap_{j} G_{x_{j}, s+n \epsilon}$. Then, $G_{x_{i}, s+(2 n+1) \epsilon} \subset K \subset G_{x_{i}, s+n \epsilon}$ for all $i$. By definition, $\sum_{i} a_{i} \mathbb{1}_{h_{i} G_{x_{i}, s}^{\prime}}$ is mapped to $\sum_{i} a_{i} \frac{\mu_{G^{\prime}}\left(G_{x_{i}, s}^{\prime}\right)}{\mu_{G}\left(G_{x_{i}, s}\right)} \mathbb{1}_{\gamma h_{i} G_{x_{i}, s}}$. By (a),

$$
\sum_{i} a_{i} \frac{\mu_{G^{\prime}}\left(G_{x_{i}, s}^{\prime}\right)}{\mu_{G}\left(G_{x_{i}, s}\right)} \mathbb{1}_{\gamma h_{i} G_{x_{i}, s}} \equiv \sum_{i} a_{i} \frac{\mu_{G^{\prime}}\left(G_{x_{i}, s}^{\prime}\right)}{\mu_{G}\left(G_{x_{i}, s}^{\prime} K\right)} \mathbb{1}_{\gamma h_{i} G_{x_{i}, s}^{\prime} K} \quad \bmod [\mathcal{H}, \mathcal{H}] .
$$

Note that $\frac{\mu_{G^{\prime}}\left(G_{x_{i}, s}^{\prime}\right)}{\mu_{G}\left(G_{x_{i}, s}^{\prime} K\right)}=\frac{\mu_{G^{\prime}}\left(G^{\prime} \cap K\right)}{\mu_{G}(K)}$ for all $i$. Moreover, we have $\sum_{i} a_{i} \mathbb{1}_{\gamma h_{i} G_{x_{i}, s}^{\prime} K}=$ 0 as $\sum_{i} a_{i} \mathbb{1}_{h_{i} G_{x_{i}, s}^{\prime}}=0$. Therefore, under the map in the proposition, the image of $\sum_{i} a_{i} \mathbb{1}_{h_{i} G_{x_{i}, s}^{\prime}}$ equals 0. Hence it gives a well-defined map from $i_{\gamma, s}: \mathcal{H}_{t, s}^{G^{\prime}, b} \rightarrow \overline{\mathcal{H}}_{s}^{G}$.

Now let $\epsilon^{\prime}=s^{\prime}-t$. Let $f \in \mathcal{H}_{t, s}^{G^{\prime}, b} \subset \mathcal{H}_{t, s^{\prime}}^{G^{\prime}, b}$. We show that $i_{\gamma, s}(f)=i_{\gamma, s^{\prime}}(f)$. 
Suppose that $f=\sum_{i} a_{i} \mathbb{1}_{h_{i} G_{x_{i}, s}^{\prime}}$. We choose an open compact subgroup $K$ such that there exists $n, n^{\prime} \in \mathbb{N}$ with $G_{x_{i}, s+(2 n+1) \epsilon} \subset K \subset G_{x_{i}, s+n \epsilon}$ and $G_{x_{i}, s^{\prime}+\left(2 n^{\prime}+1\right) \epsilon^{\prime}} \subset$ $K \subset G_{x_{i}, s^{\prime}+n^{\prime} \epsilon^{\prime}}$ for all $i$. By (a), we have that

$$
i_{\gamma, s}(f) \equiv \sum_{i} a_{i} \frac{\mu_{G^{\prime}}\left(G^{\prime} \cap K\right)}{\mu_{G}(K)} \mathbb{1}_{\gamma h_{i} G_{x_{i}, s}^{\prime} K} \quad \bmod [\mathcal{H}, \mathcal{H}] .
$$

We may write $f$ as $f=\sum_{i} \sum_{g^{\prime} \in G_{x_{i}, s}^{\prime} / G_{x_{i}, s^{\prime}}^{\prime}} a_{i} \mathbb{1}_{h_{i} g^{\prime} G_{x_{i}, s}^{\prime}} \in \mathcal{H}_{t, s^{\prime}}^{G^{\prime}, b}$. Then by (a), we have

$$
\begin{aligned}
i_{\gamma, s^{\prime}}(f) & \equiv \sum_{i} \sum_{g^{\prime} \in G_{x_{i}, s}^{\prime} / G_{x_{i}, s^{\prime}}^{\prime}} a_{i} \frac{\mu_{G^{\prime}}\left(G^{\prime} \cap K\right)}{\mu_{G}(K)} \mathbb{1}_{\gamma h_{i} g^{\prime} G_{x_{i}, s^{\prime}}^{\prime} K} K \\
& \equiv \sum_{i} a_{i} \frac{\mu_{G^{\prime}}\left(G^{\prime} \cap K\right)}{\mu_{G}(K)} \mathbb{1}_{\gamma h_{i} G_{x_{i}, s}^{\prime} K} \equiv i_{\gamma, s}(f) \quad \bmod [\mathcal{H}, \mathcal{H}] .
\end{aligned}
$$

It remains to show that the map $i_{\gamma, s}: \mathcal{H}_{t, s}^{G^{\prime}, b} \rightarrow \overline{\mathcal{H}}_{s}^{G}$ factors through $\overline{\mathcal{H}}_{t, s}^{G^{\prime}, b}$.

Let $f \in \mathcal{H}_{t, s}^{G^{\prime}, b} \cap\left[\mathcal{H}^{G^{\prime}}, \mathcal{H}^{G^{\prime}}\right]$. Then by definition, the support of $f$ is contained in the $G^{\prime}$-domain $G_{t^{+}}^{\prime}$. By Lemma 1.2.2 $f=\sum_{i}\left(f_{i}-{ }^{g_{i}} f_{i}\right)$, where $f_{i} \in \mathcal{H}\left(G^{\prime}\right)$ with support in $G_{t^{+}}^{\prime}$ and $g_{i} \in G^{\prime}$. Let $s^{\prime} \in \tilde{\mathbb{R}}$ with $s^{\prime} \geq s$ and that $f_{i} \in \mathcal{H}_{t, s^{\prime}}^{G^{\prime}, b}$ for all $i$. Then $i_{\gamma, s}(f)=i_{\gamma, s^{\prime}}(f)$. It remains to prove that for any $f^{\prime} \in \mathcal{H}_{t, s^{\prime}}^{G^{\prime}, b}$ and $g \in G^{\prime}$, we have $i_{\gamma, s^{\prime}}\left(f^{\prime}\right)=i_{\gamma, s^{\prime}}\left(g^{\prime}\right)$.

It suffices to consider the case where $f=\mathbb{1}_{h G_{x, s^{\prime}}^{\prime}}$, where $x \in \mathcal{B}(\gamma)$ and $h \in$ $G_{x, t}^{\prime} \cap G_{t^{+}}^{\prime}$. By definition,

$$
\begin{aligned}
i_{\gamma, s^{\prime}}\left(\mathbb{1}_{g\left(h G_{x, s^{\prime}}^{\prime}\right)}\right) & \equiv i_{\gamma, s^{\prime}}\left(\mathbb{1}_{g h g^{-1} G_{g x, s^{\prime}}^{\prime}}\right) \equiv \frac{\mu_{G^{\prime}}\left(G_{g x, s^{\prime}}^{\prime}\right)}{\mu_{G}\left(G_{g x, s^{\prime}}\right)} \mathbb{1}_{\gamma g h g^{-1} G_{g x, s^{\prime}}} \\
& \equiv \frac{\mu_{G^{\prime}}\left(G_{x, s^{\prime}}^{\prime}\right)}{\mu_{G}\left(G_{x, s^{\prime}}\right)} \mathbb{1}_{\gamma h G_{x, s^{\prime}}} \equiv i_{\gamma, s^{\prime}}\left(\mathbb{1}_{h G_{x, s^{\prime}}^{\prime}}\right) \bmod [\mathcal{H}, \mathcal{H}]
\end{aligned}
$$

This finishes the proof.

Theorem 3.4.3. Let $\gamma \in G^{\mathrm{rig}} \cap G^{s s}$ with a good product $\gamma=\gamma_{z} \gamma_{b_{1}} \cdots \gamma_{b_{k}} \gamma_{r^{+}}$, where each $\gamma_{b_{i}}$ is $\mathbb{G}$-good of depth $b_{i}$ and $b_{1}<b_{2}<\cdots<b_{k} \leq r$. Then the map $\mathbb{1}_{h H_{x, r}^{\gamma}} \mapsto \frac{\left.\mu_{H^{\gamma}\left(H_{x, r^{+}}^{\gamma}\right)}\right)}{\mu_{G}\left(G_{x, r^{+}}\right)} \mathbb{1}_{\gamma_{\leq r} h G_{x, r}+}$ for $x \in \mathcal{B}\left(\gamma_{\leq r}\right)$ and $h \in H_{x, r}^{\gamma} \cap H_{r^{+}}^{\gamma}$ induces a well-defined map

$$
\bar{i}_{\gamma, r^{+}}: \overline{\mathcal{H}}_{r, r^{+}}^{H^{\gamma}, b} \rightarrow \overline{\mathcal{H}}_{b_{1}, r^{+}}^{G}\left(\gamma_{z}\right) .
$$

In particular, the map $\bar{i}_{\gamma, r^{+}}$is independent of the good product expression $\gamma=$ $\gamma_{z} \gamma_{b_{1}} \cdots \gamma_{b_{k}} \gamma_{r^{+}}$.

Proof. Without loss of generality, we may assume $\gamma=\gamma_{\leq r}$. Set $\mathbb{H}^{i}=C_{\mathbb{G}}\left(\gamma_{z} \gamma_{b_{1}} \cdots \gamma_{b_{i}}\right)$. Then by Proposition 3.4.1, we have well-defined maps

$$
\begin{gathered}
\bar{i}_{\gamma_{b_{k}, r^{+}} H^{k-1}}: \overline{\mathcal{H}}_{b_{k}, r^{+}}^{H^{\gamma}, b} \rightarrow \overline{\mathcal{H}}_{b_{k}, r^{+}}^{H^{k-1},} \\
\bar{i}_{\gamma_{b_{k-1}, r^{+}} H^{k-2}}: \overline{\mathcal{H}}_{b_{k-1}, r^{+}}^{H^{k-1}, b} \rightarrow \overline{\mathcal{H}}_{b_{k-1}, r^{+}}^{H^{k-2}}, \\
\ldots \\
\bar{i}_{\gamma_{b_{1}, r^{+}}}^{G}: \overline{\mathcal{H}}_{b_{1}, r^{+}}^{H^{1}, b} \rightarrow \overline{\mathcal{H}}_{b_{1}, r^{+}}^{G}\left(\gamma_{z}\right) .
\end{gathered}
$$


As $b_{i+1}<b_{i}$ for any $i$, we have that $\overline{\mathcal{H}}_{b_{i+1}, r^{+}}^{H^{i}} \subset \overline{\mathcal{H}}_{b_{i}, r^{+}}^{H^{i}, b}$. Set $\bar{i}_{\gamma_{b_{1}}, \gamma_{b_{2}}, \cdots, \gamma_{b_{k}}, r^{+}}=$ $\bar{i}_{\gamma_{b_{1}}, r^{+}}^{G} \circ \cdots \circ \bar{i}_{\gamma_{b_{k}, r^{+}} H^{k-1}}^{G}$. This is a well-defined map from $\overline{\mathcal{H}}_{b_{k}, r^{+}}^{H^{\gamma}, b}$ to $\overline{\mathcal{H}}_{b_{1}, r^{+}}^{G}$. In particular, we have $\bar{i}_{\gamma, r^{+}}: \overline{\mathcal{H}}_{r, r^{+}}^{H^{\gamma}, b} \rightarrow \overline{\mathcal{H}}_{b_{1}, r^{+}}^{G}$.

For any $x \in \mathcal{B}\left(\gamma_{\leq r}\right)$ and $h \in H_{x, r}^{\gamma} \cap H_{r^{+}}^{\gamma}$, we have

$$
\begin{aligned}
\bar{i}_{\gamma_{b_{1}}, \gamma_{b_{2}}, \cdots, \gamma_{b_{k}}, r^{+}}\left(\mathbb{1}_{h H_{x, r^{+}}^{\gamma}}\right) \\
\equiv \bar{i}_{\gamma_{b_{1}}, \gamma_{b_{2}}, \cdots, \gamma_{b_{k-1}, r^{+}}}\left(\frac{\mu_{H^{\gamma}}\left(H_{x, r^{+}}^{\gamma}\right)}{\mu_{H^{k-1}}\left(H_{x, r^{+}}^{k-1}\right)} \mathbb{1}_{\gamma_{b_{k}} h H_{x, r^{+}}^{k-1}}\right) \\
\equiv \bar{i}_{\gamma_{b_{1}}, \gamma_{b_{2}}, \cdots, \gamma_{b_{k-2}, r^{+}}}\left(\frac{\mu_{H^{\gamma}}\left(H_{x, r^{+}}^{\gamma}\right)}{\mu_{H^{k-1}}\left(H_{x, r^{+}}^{k-1}\right)} \frac{\mu_{H^{k-1}}\left(H_{x, r^{+}}^{k-1}\right)}{\left.\mu_{H^{k-2}\left(H_{x, r^{+}}^{k-2}\right)} \mathbb{1}_{\gamma_{b_{k-1}} \gamma_{b_{k}} h H_{x, r^{+}}^{k-2}}\right)}\right. \\
\equiv \cdots \\
\equiv \frac{\mu_{H^{\gamma}}\left(H_{x, r^{+}}^{\gamma}\right)}{\mu_{H^{k-1}}\left(H_{x, r^{+}}^{k-1}\right)} \frac{\mu_{H^{k-1}}\left(H_{x, r^{+}}^{k-1}\right)}{\mu_{H^{k-2}}\left(H_{x, r^{+}}^{k-2}\right)} \cdots \frac{\mu_{H^{1}}\left(H_{x, r^{+}}^{1}\right)}{\mu_{G}\left(G_{x, r^{+}}\right)} \mathbb{1}_{\gamma h G_{x, r^{+}}} \\
\equiv \frac{\mu_{H^{\gamma}}\left(H_{x, r^{+}}^{\gamma}\right)}{\mu_{G}\left(G_{x, r^{+}}\right)} \mathbb{1}_{\gamma h G_{x, r^{+}}} \bmod [\mathcal{H}, \mathcal{H}] .
\end{aligned}
$$

Thus the map $\bar{i}_{\gamma_{b_{1}}, \gamma_{b_{2}}, \cdots, \gamma_{b_{k}}, r^{+}}$only depends on $\gamma$. This finishes the proof.

Proof of Theorem 3.1.2. We have shown in Corollary 3.3.3 that

$$
\overline{\mathcal{H}}_{r^{+}}^{\mathrm{rig}}=\bigoplus_{[\gamma]_{\mathrm{g}} \in \mathcal{S}_{r}^{\mathrm{g}}} \overline{\mathcal{H}}_{[\gamma]_{\mathrm{g}}}^{G, \mathrm{~b}} .
$$

Let $\gamma_{z} \gamma_{b_{1}}$ be a $\mathbb{G}$-good mod center element and $\mathbb{1}_{g G_{y, r}} \in \mathcal{H}_{\left[\gamma_{z} \gamma_{b_{1}}\right]_{\mathrm{g}}}^{G, b}$. By Proposition 3.4.1, $\bar{i}_{\gamma_{b_{1}, r^{+}}}: \overline{\mathcal{H}}_{b_{1}, r^{+}}^{H^{1}, b} \rightarrow \overline{\mathcal{H}}_{\left[\gamma_{z} \gamma_{\left.b_{1}\right]_{g}, r^{+}}\right.}^{G}$, where $H^{1}=C_{G}\left(\gamma_{z} \gamma_{b_{1}}\right)$, is a well defined surjective map. Write $g=\gamma_{z} \gamma_{b_{1}} u_{1}$ where $u_{1} \in H_{y, b_{1}}^{1} \cap H_{b_{1}^{+}}^{1}$. Then, $\mathbb{1}_{u_{1} H_{y, r^{+}}^{1}} \equiv \sum_{j} c_{j} \mathbb{1}_{\gamma_{b_{2 j}} u_{2 j} H_{y_{2 j}, r^{+}}^{1}}$ for some $H^{1}$-good elements $\gamma_{b_{2 j}}$ of depth $b_{2 j}>b_{1}$, $y_{2 j} \in \mathcal{B}\left(H^{2 j}\right)$ where $H^{2 j}=C_{H^{1}}\left(\gamma_{b_{2 j}}\right), c_{j} \in \mathbb{Z}\left[\frac{1}{p}\right]$ and $u_{2 j} \in H_{y_{2 j}, b_{2 j}}^{2 j} \cap H_{b_{2 j}^{+}}^{2 j}$. Note that there are constants $c_{j}^{\prime} \in \mathbb{Z}\left[\frac{1}{p}\right]$ such that

$$
\sum_{j} c_{j}^{\prime} \cdot \mathbb{1}_{\gamma_{b_{2 j}} u_{2 j} H_{y_{2 j}, r+}^{1}} \stackrel{\bar{i}_{\gamma_{b_{1}, r}+}^{\longrightarrow}}{\longrightarrow} \sum_{j} c_{j} \cdot \mathbb{1}_{\gamma_{z} \gamma_{b_{1}} \gamma_{b_{2 j}} u_{2 j} G_{y_{2 j}, r}} \equiv \mathbb{1}_{g G_{y, r^{+}}} .
$$

Repeating the process to each summand, $\mathbb{1}_{u_{2 j} H_{y, r^{+}}^{2 j}} \equiv \sum_{k} c_{k} \cdot \mathbb{1}_{\gamma_{b_{2 j k} u_{2 j k} H_{y_{2 j k}, r^{+}}^{2 j}}}$ for some $H^{2 j}$-good elements $\gamma_{b_{2 j k}}$ of depth $b_{2 j k}>b_{2 j}, y_{2 j k} \in \mathcal{B}\left(H^{2 j k}\right)$ where $H^{2 j k}=C_{H^{2 j}}\left(\gamma_{b_{2 j k}}\right)$ and $u_{2 j k} \in H_{y_{2 j k}, b_{2 j k}}^{2 j k} \cap H_{b_{2 j k}^{+}}^{2 j k}$. Now

$$
\begin{aligned}
& \sum_{k} \sum_{j} c_{j k} \cdot \mathbb{1}_{\gamma_{b_{2 j k}} u_{2 j k} H_{y_{2 j k}, r^{+}}^{1}} \stackrel{\sum_{j} \bar{i}_{\gamma_{b_{2 j}}, r^{+}}}{\longrightarrow} \sum_{j} c_{j}^{\prime} \mathbb{1}_{\gamma_{b_{2 j}} \gamma_{b_{2 j k}} u_{2 j} H_{y_{2 j}, r^{+}}^{1}} \\
& \stackrel{\bar{i}_{\gamma_{b_{1}}, r+}}{\longrightarrow} \sum_{j} c_{j} \mathbb{1}_{\gamma_{z} \gamma_{b_{1}} \gamma_{b_{2 j}} \gamma_{b_{2 j k}} u_{2 j} G_{y_{2 j}, r^{+}}} \equiv \mathbb{1}_{g G_{y, r}+} \quad \bmod [\mathcal{H}, \mathcal{H}] .
\end{aligned}
$$


Setting $b_{i}$ to be the min of depths appearing in summands in each $i$ th step. One can repeat the process until $b_{i}>r$. These are finitely many steps since $b_{i}$ forms an increasing discrete sequence. Now, we proved that

$$
\overline{\mathcal{H}}_{r^{+}}^{\text {rig }} \subset \sum_{[\gamma] \in \mathcal{S}_{r}} \bar{i}_{\gamma, r^{+}}\left(\overline{\mathcal{H}}_{r, r^{+}}^{C_{G}(\gamma), b}\right) \subset \sum_{[\gamma] \in \mathcal{S}_{r}} \overline{\mathcal{H}}_{[\gamma]}^{G, b} \subset \sum_{[\gamma] \in \mathcal{S}_{r}} \overline{\mathcal{H}}_{r^{+}}\left({ }^{G}\left(\gamma H_{r^{+}}^{\gamma}\right)\right) \subset \overline{\mathcal{H}}_{r^{+}}^{\text {rig }} .
$$

Therefore all the inclusions above are in fact equalities. Also

$$
\overline{\mathcal{H}}^{\text {rig }}=\sum_{[\gamma] \in \mathcal{S}_{r}} \overline{\mathcal{H}}_{r^{+}}\left({ }^{G}\left(\gamma H_{r^{+}}^{\gamma}\right)\right) .
$$

By Lemma 1.2.3, this is a direct sum. Moreover, for any $[\gamma] \in \mathcal{S}_{r}$, we have

$$
\bar{i}_{\gamma, r^{+}}\left(\overline{\mathcal{H}}_{r, r^{+}}^{H^{\gamma}, b}\right)=\overline{\mathcal{H}}_{[\gamma]}^{G, b}=\overline{\mathcal{H}}_{r^{+}}\left({ }^{G}\left(\gamma_{\leq r} H_{r^{+}}^{\gamma}\right)\right) .
$$

As shown in the proof, we have the following description of $\overline{\mathcal{H}}_{r^{+}}\left({ }^{G}\left(\gamma H_{r+}^{\gamma}\right)\right)$.

Corollary 3.4.4. Let $\bar{i}_{\gamma, r^{+}}$be as in Theorem 3.4.3. Then, $\bar{i}_{\gamma, r^{+}}\left(\overline{\mathcal{H}}_{r, r^{+}}^{H^{\gamma}, b}\right)=\overline{\mathcal{H}}_{[\gamma]}^{G, b}$.

\section{JoRDAN DECOMPOSITION OF $\overline{\mathcal{H}}_{r+}^{\text {rig }}$}

4.1. The cosets $I_{r}^{u}$ and $I_{r}^{d}$. Following [8,9, we set

$$
I_{r}(G)=\left\{\left(F^{*}, X\right) ; F^{*} \text { is a generalized } r \text {-facet of } G, X \in G_{F^{*}} / G_{F^{*}}^{+}\right\},
$$

$I_{r}^{u}(G)=\left\{\left(F^{*}, X\right) \in I_{r}(G) ; X=u G_{F^{*}}^{+}\right.$for some unipotent element $\left.u \in G_{F^{*}}\right\}$.

By [2, Corollary 3.7.10], $I_{r}^{u}(G)=\left\{\left(F^{*}, X\right) \in I_{r}(G) ; X \subset G_{r^{+}}\right\}$.

By [8, Definition 5.3.4] and [9, §4.4], under Hypotheses (DB), to each pair $\left(F^{*}, X\right) \in I_{r}^{u}(G)$, there exists a unique unipotent conjugacy class of minimal dimension which intersects $X$. We denote this unipotent conjugacy class by $\mathcal{O}\left(F^{*}, X\right)$.

Finally, we define the distinguished cosets $I_{r}^{d}(G) \subset I_{r}^{u}(G)$ as in [8, Definition 5.5.1] and the equivalence relation $\sim$ as in [8, Definition 3.6.2]. By [8, Theorem 5.6.1], under Hypotheses (DB), the map $\left(F^{*}, X\right) \mapsto \mathcal{O}\left(F^{*}, X\right)$ gives a bijection between $I_{r}^{d}(G) / \sim$ and the set $C l^{u}(G)$ of unipotent conjugacy classes of $G$.

We first prove the following.

Proposition 4.1.1. Suppose Hypotheses (DB) and [5 hold. Then $\overline{\mathcal{H}}_{[1]}^{G, b}$ is a free $\mathbb{Z}\left[\frac{1}{p}\right]$-module with basis $\mathbb{1}_{\left(F^{*}, X\right)}$, where $\left(F^{*}, X\right)$ runs over representatives in $I_{r}^{d}(G) / \sim$ and $\mathbb{1}_{\left(F^{*}, X\right)}$ is the characteristic function $\mathbb{1}_{X}$ supported on $X$.

We adapt the strategy of $[9, \S 2]$. While the invariant distributions (with complex coefficients) are considered in [9], here we consider the cocenter of $\mathcal{H}$ and need to work with coefficients in $\mathbb{Z}\left[\frac{1}{p}\right]$.

Lemma 4.1.2. Let $\mathcal{O}$ be a unipotent conjugacy class of $G$ and $\left(F_{1}^{*}, X_{1}\right),\left(F_{2}^{*}, X_{2}\right) \in$ $I_{r}^{u}(G)$ are two pairs associated to $\mathcal{O}$ (i.e., $\left.\mathcal{O}=\mathcal{O}\left(F_{1}^{*}, X_{1}\right)=\mathcal{O}\left(F_{2}^{*}, X_{2}\right)\right)$ such that $F_{2}^{*} \subset \overline{F_{1}^{*}}$ and $X_{2} \subset X_{1}$. Then in $\overline{\mathcal{H}}$, we have

$$
\mathbb{1}_{X_{1}} \in p^{n} \mathbb{1}_{X_{2}}+\sum_{\left(F^{*}, X\right) \in I_{r}^{u}(G) \text { with } \mathcal{O}\left(F^{*}, X\right)>\mathcal{O}} \mathbb{Z}\left[\frac{1}{p}\right] \mathbb{1}_{X}+[\mathcal{H}, \mathcal{H}] \quad \text { for some } n \in \mathbb{N} \text {. }
$$

Here $\leq$ is the partial order on the set of unipotent conjugacy classes of G given by the closure relation. 
Proof. We follow the argument in [9, Lemma 2.6.2], almost verbatim. We have that

$$
G_{F_{2}^{*}}^{+} \subset G_{F_{1}^{*}}^{+} \subset G_{F_{1}^{*}} \subset G_{F_{2}^{*}} \text {. }
$$

We write $\mathbb{1}_{X_{1}}$ as

$$
\mathbb{1}_{X_{1}}=\sum_{Y \in X_{1} / G_{F_{2}^{*}}^{+}} \mathbb{1}_{Y}
$$

Let $Y \in X_{1} / G_{F_{2}^{*}}^{+}$. Then $Y \subset X_{1} \subset G_{r^{+}}$. By [8, Corollary 5.2.5], we have $\mathcal{O}\left(F_{2}^{*}, Y\right) \geq \mathcal{O}$. The case where $\mathcal{O}\left(F_{2}^{*}, Y\right)>\mathcal{O}$ is obvious. It remains to consider the case where $\mathcal{O}\left(F_{2}^{*}, Y\right)=\mathcal{O}$. By [8, Lemma 3.2.17], there exists $x \in F_{1}^{*}$ so that $G_{x} \subset \operatorname{Stab}_{G}\left(F_{2}^{*}\right)$. By [8, Corollary 5.2.3], we have $Y={ }^{g} X_{2}$ for some $g \in G_{x}^{+}$. In particular, $\mathbb{1}_{Y} \equiv \mathbb{1}_{X_{2}} \bmod [\mathcal{H}, \mathcal{H}]$. Set $\Gamma=\left\{Y \in X_{1} / G_{F_{2}^{*}}^{+} ; \mathcal{O}\left(F_{2}^{*}, Y\right)=\mathcal{O}\right\}$. Then $G_{x}^{+}$acts transitively on $\Gamma$. Since $G_{x}^{+}$is a pro- $p$ group, the cardinality of $\Gamma$ is a power of $p$. The statement is proved.

The following results follow easily from Lemma 4.1.2 and the definition of $\sim$ (see the proof of [9, Lemma 2.6.5]).

Corollary 4.1.3. Let $\left(F_{1}^{*}, X_{1}\right),\left(F_{2}^{*}, X_{2}\right) \in I_{r}^{d}(G)$ with $\left(F_{1}^{*}, X_{1}\right) \sim\left(F_{2}^{*}, X_{2}\right)$. Then $\mathbb{1}_{X_{1}} \in p^{n} \mathbb{1}_{X_{2}}+\sum_{\left(F^{*}, X\right) \in I_{r}^{u}(G) \text { with } \mathcal{O}\left(F^{*}, X\right)>\mathcal{O}} \mathbb{Z}\left[\frac{1}{p}\right] \mathbb{1}_{X}+[\mathcal{H}, \mathcal{H}] \quad$ for some $n \in \mathbb{Z}$.

Proof of Proposition 4.1.1. Note that $\overline{\mathcal{H}}_{[1]}^{G, b}$ is spanned by $\mathbb{1}_{\left(F^{*}, X\right)}$ for $\left(F^{*}, X\right) \in$ $I_{r}^{u}(G)$. By definition, for any $\left(F^{*}, X\right) \in I_{r}^{u}(G)$, there exists $\left(F_{1}^{*}, X_{1}\right) \in I_{r}^{d}(G)$ such that $F^{*} \subset \overline{F_{1}^{*}}$ and $X \subset X_{1}$. By Lemma 4.1.2, given a unipotent conjugacy class $\mathcal{O}$, for any $\left(F^{*}, X\right) \in I_{r}^{u}(G)$ with $\mathcal{O}\left(F^{*}, X\right)=\mathcal{O}$, the element $\mathbb{1}_{\left(F^{*}, X\right)}$ in $\overline{\mathcal{H}}$ is contained in the span of $\mathbb{1}_{\left(F_{1}^{*}, X_{1}\right)}$, where $\left(F_{1}^{*}, X_{1}\right) \in I_{r}^{d}(G)$ with $\mathcal{O}\left(F_{1}^{*}, X_{1}\right)=\mathcal{O}$, and $\left(F^{\prime *}, X^{\prime}\right) \in I_{r}^{u}(G)$ where $\mathcal{O}\left(F^{\prime *}, X^{\prime}\right)>\mathcal{O}$. Here, we denote $\mathbb{1}_{X}$ by $\mathbb{1}_{\left(F^{*}, X\right)}$ for clarity.

By Corollary 4.1.3, it suffices to use any representative $\left(F_{1}^{*}, X_{1}\right) \in I_{r}^{d}(G) / \sim$ with $\mathcal{O}\left(F_{1}^{*}, X_{1}\right)=\mathcal{O}$ instead of all the distinguished cosets associated to $\mathcal{O}$.

By Hypotheses (DB), there are only finitely many unipotent conjugacy classes. Hence by induction, $\mathbb{1}_{\left(F^{*}, X\right)}$ is spanned by $\mathbb{1}_{\left(F^{\prime *}, X^{\prime}\right)}$, where $\left(F^{\prime *}, X^{\prime}\right)$ runs over representatives in $I_{r}^{d}(G) / \sim$ with $\mathcal{O}\left(F^{\prime *}, X^{\prime}\right) \geq \mathcal{O}$. In particular, $\overline{\mathcal{H}}_{[1]}^{G, \mathrm{~b}}$ is spanned by $\mathbb{1}_{\left(F^{\prime *}, X^{\prime}\right)}$, where $\left(F^{* *}, X^{\prime}\right)$ runs over representatives in $I_{r}^{d}(G) / \sim$.

Now we choose a set of representatives $\left(F^{\prime *}, X^{\prime}\right)$ of $I_{r}^{d}(G) / \sim$. It remains to show that the elements $\mathbb{1}_{\left(F^{\prime *}, X^{\prime}\right)}$ are linearly independent over $\mathbb{Z}\left[\frac{1}{p}\right]$. Suppose that $\sum a_{\left(F^{\prime *}, X^{\prime}\right)} \mathbb{1}_{\left(F^{\prime *}, X^{\prime}\right)} \equiv 0$ in $\overline{\mathcal{H}}$ with $a_{\left(F^{\prime *}, X^{\prime}\right)} \in \mathbb{Z}\left[\frac{1}{p}\right] \subset \mathbb{C}$. We regard $\sum a_{\left(F^{\prime *}, X^{\prime}\right)} \mathbb{1}_{\left(F^{\prime *}, X^{\prime}\right)}$ as the zero element in $\overline{\mathcal{H}}_{\mathbb{C}}$. Suppose that not all the coefficients $a_{\left(F^{\prime *}, X^{\prime}\right)}$ are 0 . Let $\mathcal{O}$ be a minimal unipotent conjugacy class such that $\mathcal{O}=\mathcal{O}\left(F_{1}^{\prime *}, X_{1}^{\prime}\right)$ for some $\left(F_{1}^{\prime *}, X_{1}^{\prime}\right)$ with $a_{\left(F_{1}^{\prime *}, X_{1}^{\prime}\right)} \neq 0$. By the minimality assumption, for any other representative $\left(F^{\prime *}, X^{\prime}\right)$ in our chosen set, we have $a_{\left(F^{\prime *}, X^{\prime}\right)}=0$ or $\mathcal{O} \cap X^{\prime}=\emptyset$. For $u \in \mathcal{O}$, we have

$$
\begin{aligned}
0 & =O_{u}\left(a_{\left(F_{1}^{\prime *}, X_{1}^{\prime}\right)} \mathbb{1}_{\left(F_{1}^{\prime *}, X_{1}^{\prime}\right)}\right)+\sum_{\left(F^{\prime *}, X^{\prime}\right) \neq\left(F_{1}^{\prime *}, X_{1}^{\prime}\right)} O_{u}\left(a_{\left(F^{\prime *}, X^{\prime}\right)} \mathbb{1}_{\left(F^{\prime *}, X^{\prime}\right)}\right) \\
& =a_{\left(F_{1}^{\prime *}, X_{1}^{\prime}\right)} O_{u}\left(\mathbb{1}_{\left(F_{1}^{\prime *}, X_{1}^{\prime}\right)}\right) .
\end{aligned}
$$


This is a contradiction as $a_{\left(F_{1}^{\prime *}, X_{1}^{\prime}\right)} \neq 0$ and $O_{u}\left(\mathbb{1}_{\left(F_{1}^{\prime *}, X_{1}^{\prime}\right)}\right)$ is a nonzero number in $\mathbb{C}$. Hence the image of $\mathbb{1}_{\left(F^{\prime *}, X^{\prime}\right)}$ in $\overline{\mathcal{H}}$ for any set of representatives $\left(F^{*}, X^{\prime}\right)$ of $I_{r}^{d}(G) / \sim$ are linearly independent.

4.2. Unipotent orbits in the group $H^{\gamma}$. Now we consider $\overline{\mathcal{H}}_{[\gamma]}^{G, b}$ for arbitrary $[\gamma] \in \mathcal{S}_{r}$. Set $\mathbb{H}=\mathbb{H}^{\gamma}$. Note that $\mathbb{H}$ is not connected in general. The cosets we consider in this situation are the distinguished cosets $I_{r}^{d}(H):=I_{r}^{d}\left(H^{\circ}\right)$, but there are extra equivalence relations that we need to take into account. The equivalence relation $\tilde{\sim}$ on $I_{r}^{d}(H)$ is generated by the equivalence relation $\sim$ on $I^{d}\left(H^{\circ}\right)$ in 8 , Definition 3.6.2] and the relation $\left(F^{*}, X\right) \tilde{\sim}\left({ }^{h} F^{*},{ }^{h} X\right)$ for $h \in H$. In other words, the group $H / H^{\circ}$ acts naturally on $I_{r}^{d}\left(H^{\circ}\right) / \sim$ and the quotient set is $I_{r}^{d}(H) / \tilde{\sim}$.

On the other hand, let $C l^{u}(H)$ be the set of unipotent conjugacy classes of $H$ and let $C l^{u}\left(H^{\circ}\right)$ be the set of unipotent conjugacy classes of $H^{\circ}$. Under Hypothesis 2 , the natural map $C l^{u}\left(H^{\circ}\right) \rightarrow C l^{u}(H)$ is surjective. The group $H / H^{\circ}$ acts naturally on $C l^{u}\left(H^{\circ}\right)$ and the quotient set is $C l^{u}(H)$.

It is easy to see that the map $I_{r}^{d}\left(H^{\circ}\right) / \sim \rightarrow C l^{u}\left(H^{\circ}\right)$ given by $\left(F^{*}, X\right) \mapsto$ $\mathcal{O}\left(F^{*}, X\right)$ is $H / H^{\circ}$-equivariant. Thus it leads to a map $I_{r}^{d}(H) / \tilde{\sim} \rightarrow C l^{u}(H)$. Combining this with the result in [9, §4.4], under Hypotheses (DB) and 2, this map is bijective.

Now we come to the main result of this section.

Theorem 4.2.1. Suppose Hypotheses (DB), (A), (C), 2, and [5 hold. Then for any $[\gamma] \in \mathcal{S}_{r}$,

(1) $\overline{\mathcal{H}}_{r, r^{+}}^{H^{\gamma}, b}$ is a free $\mathbb{Z}\left[\frac{1}{p}\right]$-module with basis $\mathbb{1}_{\left(F^{*}, X\right)}$, where $\left(F^{*}, X\right)$ runs over representatives in $I_{r}^{d}\left(H^{\gamma}\right) / \tilde{\sim}$.

(2) The map $\bar{i}_{\gamma, r^{+}}: \overline{\mathcal{H}}_{r, r^{+}}^{H^{\gamma}, b} \rightarrow \overline{\mathcal{H}}_{[\gamma]}^{G, b}$ defined in Theorem 3.4.3 is a $\mathbb{Z}\left[\frac{1}{p}\right]$-linear isomorphism.

Proof. Without loss of generality, we assume $\gamma=\gamma_{\leq r}$.

By definition, if $h \in H^{\gamma}$, then for any $\left(F^{*}, X\right) \in I_{r}^{d}\left(H^{\gamma}\right)$, we have $\mathbb{1}_{\left(F^{*}, X\right)} \equiv$ $\mathbb{1}_{\left({ }^{h} F^{*}, h_{X}\right)} \bmod \left[\mathcal{H}^{H^{\gamma}}, \mathcal{H}^{H^{\gamma}}\right]$. We choose a set $A$ of representatives $\left(F^{*}, X\right)$ in $I_{r}^{d}\left(H^{\gamma}\right) / \tilde{\sim}$. By Proposition 4.1.1 applied to $H^{\gamma}, \overline{\mathcal{H}}_{r, r^{+}}^{H^{\gamma}}$ is spanned by $\mathbb{1}_{\left(F^{*}, X\right)}$. By Corollary [3.4.4, $\bar{i}_{\gamma, r+}\left(\overline{\mathcal{H}}_{r, r^{+}}^{H^{\gamma}, b}\right)=\overline{\mathcal{H}}_{[\gamma]}^{G, b}$.

Now suppose that $\bar{i}_{\gamma, r^{+}}\left(\sum_{\left(F^{*}, X\right) \in A} a_{\left(F^{*}, X\right)} \mathbb{1}_{\left(F^{*}, X\right)}\right) \equiv 0$ in $\overline{\mathcal{H}}$; here $a_{\left(F^{*}, X\right)} \in$ $\mathbb{Z}\left[\frac{1}{p}\right] \subset \mathbb{C}$. We regard $\bar{i}_{\gamma, r^{+}}\left(\sum_{\left(F^{*}, X\right) \in A} a_{\left(F^{*}, X\right)} \mathbb{1}_{\left(F^{*}, X\right)}\right)$ as the zero element in $\overline{\mathcal{H}}_{\mathbb{C}}$. Suppose that not all the coefficients $a_{\left(F^{*}, X\right)}$ are 0 . Let $\mathcal{O}$ be a minimal unipotent conjugacy of $H^{\gamma}$ such that there exists $\left(F^{*}, X\right)$ in the chosen set of representatives with $\mathcal{O}\left(F^{*}, X\right)=\mathcal{O}$ and $a_{\left(F^{*}, X\right)} \neq 0$.

By Lemma 2.3.3, the map $g \mapsto \gamma g$ induces an injective map from the set of conjugacy classes of $H^{\gamma}$ to the set of conjugacy classes of $G$. Let $\mathcal{O}^{\prime}$ be the conjugacy class of $G$ that contains $\gamma \mathcal{O}$. Note that the support of $\bar{i}_{\gamma, r^{+}}\left(\mathbb{1}_{\left(F^{\prime *}, X^{\prime}\right)}\right)$ is contained in ${ }^{G} X^{\prime}$ by Lemma 2.3.2. Let $u \in \mathcal{O}$. For any element $\left(F^{*}, X^{\prime}\right)$ in our chosen set, if $\left(F^{\prime *}, X^{\prime}\right) \neq\left(F^{*}, X\right)$, then $O_{\gamma u}\left(\bar{i}_{\gamma, r^{+}}\left(a_{\left(F^{\prime *}, X^{\prime}\right)} \mathbb{1}_{\left(F^{\prime *}, X^{\prime}\right)}\right)\right)=0$. Then

$$
\begin{aligned}
0 & =O_{\gamma u}\left(\bar{i}_{\gamma, r^{+}}\left(a_{\left(F^{*}, X\right)} \mathbb{1}_{\left(F^{*}, X\right)}\right)\right)+\sum_{\left(F^{\prime *}, X^{\prime}\right) \neq\left(F^{*}, X\right)} O_{\gamma u}\left(\bar{i}_{\gamma, r^{+}}\left(a_{\left(F^{*}, X^{\prime}\right)} \mathbb{1}_{\left(F^{\prime *}, X^{\prime}\right)}\right)\right) \\
& =a_{\left(F^{*}, X\right)} O_{\gamma u}\left(\bar{i}_{\gamma, r^{+}}\left(\mathbb{1}_{\left(F^{*}, X\right)}\right)\right) .
\end{aligned}
$$


This is a contradiction as $a_{\left(F^{*}, X\right)} \neq 0$ and $O_{\gamma u}\left(\bar{i}_{\gamma, r^{+}}\left(\mathbb{1}_{\left(F^{*}, X\right)}\right)\right)$ is a nonzero number in $\mathbb{C}$. Therefore the set $\mathbb{1}_{\left(F^{*}, X\right)}$ is linearly independent and the map $\bar{i}_{\gamma, r^{+}}$ is injective.

Corollary 4.2.2. Suppose all hypotheses in $₫ 2.2$ hold. Then $\overline{\mathcal{H}}_{r+}^{\text {rig }}$ is a free $\mathbb{Z}\left[\frac{1}{p}\right]$ module. If moreover $G$ is semisimple, then the rank of $\overline{\mathcal{H}}_{r^{+}}^{\mathrm{rig}}$ is $\sum_{[\gamma] \in \mathcal{S}_{r}} \sharp C l^{u}\left(H^{\gamma}\right)$.

4.3. Application to invariant distributions. For any compact subset $X$ of $G$, we denote by $J(X)$ the space of complex-valued invariant distributions of $G$ with support in ${ }^{G} X$. Similarly, we write $\overline{\mathcal{H}}(X), \overline{\mathcal{H}}(X)_{r^{+}, \mathbb{C}}$ for $\overline{\mathcal{H}}\left({ }^{G} X\right), \overline{\mathcal{H}}\left({ }^{G} X\right)_{r^{+}, \mathbb{C}}$, etc., for simplicity. Now we discuss some application to the invariant distributions. We first recall Theorem $\mathrm{B}$ in the introduction and give a proof of it.

Theorem 4.3.1. Suppose all hypotheses in $₫ 2.2$ hold. The restriction $\left.J\left(G^{\mathrm{rig}}\right)\right|_{\mathcal{H}_{r+, \mathbb{C}}}$ has a basis given by the restriction of orbital integrals $O_{\gamma_{\leq r} u}$ to $\mathcal{H}_{r^{+}, \mathbb{C}}$, where $[\gamma] \in \mathcal{S}_{r}$, and $u$ runs over the representatives of the unipotent conjugacy classes of $H^{\gamma}$.

Proof. By Theorem 3.1.2, $\overline{\mathcal{H}}_{r^{+}, \mathbb{C}}^{\text {rig }}=\bigoplus_{[\gamma] \in \mathcal{S}_{r}} \overline{\mathcal{H}}_{r^{+}, \mathbb{C}}\left(\gamma H_{r^{+}}^{\gamma}\right)$ and each subset ${ }^{G}\left(\gamma H_{r^{+}}^{\gamma}\right)$ is a $G$-domain. We have

$$
\begin{aligned}
\left.J\left(G^{\mathrm{rig}}\right)\right|_{\mathcal{H}_{r+, \mathbb{C}}} & =\left.J\left(G^{\mathrm{rig}}\right)\right|_{\overline{\mathcal{H}}_{r+, \mathbb{C}}}=\left.J\left(G^{\mathrm{rig}}\right)\right|_{\overline{\mathcal{H}}_{r+, \mathbb{C}}^{\mathrm{rig}}}=\left.\bigoplus_{[\gamma] \in \mathcal{S}_{r}} J\left(G^{\mathrm{rig}}\right)\right|_{\overline{\mathcal{H}}_{r+, \mathbb{C}}\left(\gamma H_{r+}^{\gamma}\right)} \\
& =\bigoplus_{[\gamma] \in \mathcal{S}_{r}} \overline{\mathcal{H}}_{r^{+}, \mathbb{C}}\left(\gamma H_{r^{+}}^{\gamma}\right)^{*} .
\end{aligned}
$$

For any $[\gamma],\left[\gamma^{\prime}\right] \in \mathcal{S}_{r}$ with $\left[\gamma^{\prime}\right] \neq[\gamma]$, by Proposition 2.5 .2 we have ${ }^{G}\left(\gamma H_{r^{+}}^{\gamma}\right) \cap$ ${ }^{G}\left(\gamma^{\prime} H_{r^{+}}^{\gamma^{\prime}}\right)=\emptyset$ and hence for any unipotent element $u \in H^{\gamma}, O_{\gamma_{\leq r} u}\left(\overline{\mathcal{H}}_{r^{+}, \mathbb{C}}\left(\gamma^{\prime} H_{r^{+}}^{\gamma^{\prime}}\right)\right)=$ 0 .

Now we fix an equivalence class $[\gamma] \in \mathcal{S}_{r}$ and an element $\gamma_{\leq r}$. By the proof of Theorem 4.2.1 (2), the dimension of $\overline{\mathcal{H}}_{r^{+}, \mathbb{C}}\left(\gamma H_{r^{+}}^{\gamma}\right)$ equals the number of unipotent conjugacy classes of $H^{\gamma}$ and the orbital integrals $O_{\gamma_{\leq r} u}$, where $u$ runs over representatives of the unipotent conjugacy classes of $H^{\gamma}$, form a basis of linear functions on $\overline{\mathcal{H}}_{r^{+}, \mathbb{C}}\left(\gamma H_{r^{+}}^{\gamma}\right)$. The theorem is proved.

4.4. Finally, we explain how Theorem 4.3.1 may be applied to Howe's conjecture. In [14], Howe conjectured that for any open compact subgroup $K$ and compact subset $X$ of $G$, the restriction $\left.J(X)\right|_{\mathcal{H}_{\mathbb{C}}(G, K)}$ is finite dimensional. This is proved by Clozel [7] and by Barbasch and Moy [4. Another proof is given by the first-named author in 12 .

Following [12, we have the Newton decompositions

$$
G=\bigsqcup_{\nu \in \aleph} G(\nu) \quad \text { and } \quad G^{\mathrm{rig}}=\bigsqcup_{\nu \in \aleph ; C_{G}(\nu)=G} G(\nu),
$$

where $\aleph$ is the product of $\pi_{1}(G)$ (the Kottwitz factor) and the set of dominant rational coweights of $G$ (the Newton factor), and $G(\nu)$ is the corresponding Newton stratum defined in [12, $\S 2.2]$.

It follows from the definitions of Newton strata and $r^{+}$-equivalence that for semisimple compact-modulo-center elements, if $\gamma$ and $\gamma^{\prime}$ are $r^{+}$-equivalent mod center (for some $r$ ), then $\gamma$ and $\gamma^{\prime}$ are contained in the same Newton stratum. 
For $\nu \in \aleph$ that is central in $G$, we let $\mathcal{S}_{\nu, r}$ be the set of $r^{+}$-equivalence classes of semisimple elements in $G(\nu)$. Then we have

$$
\mathcal{S}_{r}=\bigsqcup_{\nu \in \aleph ;} \bigsqcup_{G(\nu)=G} \mathcal{S}_{\nu, r}
$$

Based on the approach of [12, the study of the restriction $\left.J(X)\right|_{\mathcal{H}_{\mathbb{C}}(G, K)}$ can be reduced to the study of $\left.J(G(\nu))\right|_{\mathcal{H}_{\mathbb{C}}\left(G, I_{n}\right)}$, where $\nu \in \aleph$ that is central in $G$ and $I_{n}$ is the $n$th congruent subgroup of an Iwahori subgroup of $G$. If $r=n-\epsilon$, where $\epsilon$ is a sufficiently small positive number, then there is an $x$ in the base alcove such that $G_{x, r^{+}}=I_{n}$ and $\overline{\mathcal{H}}_{r^{+}}=\overline{\mathcal{H}}\left(G, I_{n}\right)$. In this case, $\left.J\left(G^{\mathrm{rig}}\right)\right|_{\mathcal{H}_{r+, \mathbb{C}}}=\left.J\left(G^{\mathrm{rig}}\right)\right|_{\mathcal{H}_{\mathbb{C}}\left(G, I_{n}\right)}$.

Let $\mathcal{H}\left(G, I_{n} ; \nu\right)$ be the $\mathbb{Z}\left[\frac{1}{p}\right]$-submodule of $\mathcal{H}\left(G, I_{n}\right)$ consisting of functions with support in $G(\nu)$ and let $\overline{\mathcal{H}}\left(G, I_{n} ; \nu\right)$ be the image of $\mathcal{H}\left(G, I_{n} ; \nu\right)$ in the cocenter $\overline{\mathcal{H}}$. The main result of [12] establishes the Newton decomposition (see [12, Theorem 4.1]):

$$
\overline{\mathcal{H}}\left(G, I_{n}\right)=\bigsqcup_{\nu \in \aleph} \overline{\mathcal{H}}\left(G, I_{n} ; \nu\right) \quad \text { and } \quad \overline{\mathcal{H}}\left(G, I_{n}\right)^{\text {rig }}=\bigsqcup_{\nu \in \aleph ; C_{G}(\nu)=G} \overline{\mathcal{H}}\left(G, I_{n} ; \nu\right) .
$$

Combining it with Theorem 4.3.1, we have the following.

Theorem 4.4.1. Suppose all hypotheses in $\$ 2.2$ hold. Let $\nu \in \aleph$ such that $\nu$ is central in $G$. The restriction $\left.J(G(\nu))\right|_{\mathcal{H}_{\mathbb{C}}\left(G, I_{n}\right)}$ has a basis given by the restriction of orbital integrals $O_{\gamma_{\leq r} u}$ to $\mathcal{H}_{\mathbb{C}}\left(G, I_{n}\right)$, where $[\gamma] \in \mathcal{S}_{\nu, r}$, and $u$ runs over the representatives of the unipotent conjugacy classes of $H^{\gamma}$.

In particular, the dimension of $\left.J(G(\nu))\right|_{\mathcal{H}_{\mathbb{C}}\left(G, I_{n}\right)}$ is equal to $\sum_{[\gamma] \in \mathcal{S}_{\nu, r}} \sharp C l^{u}\left(H^{\gamma}\right)$.

This result gives an explicit basis of the finite dimensional space $\left.J(G(\nu))\right|_{\mathcal{H}_{\mathbb{C}}\left(G, I_{n}\right)}$, and thus gives a precise estimate on the dimension of $\left.J(G(\nu))\right|_{\mathcal{H}_{\mathbb{C}}\left(G, I_{n}\right)}$.

\section{ExAmples}

In this section, we give some examples to illustrate relations between the cocenter and the representations. We will work with the Hecke algebras of complex-valued functions and complex representations.

5.1. Cocenter and representations. Before we come to some concrete examples, we would like to give a brief discussion on the relation between the cocenters and the representations.

Recall that $\mathfrak{R}_{\mathbb{C}}(G)$ is the complexified Grothendieck group of smooth admissible complex representations of $G$ of finite length. Let $\mathcal{P}$ be the set of all proper parabolic subgroups of $G$. For any Levi subgroup $M$ of $G$, we denote by $\Psi(M)_{\mathbb{C}}$ the group of unramified character of $M$ over $\mathbb{C}$. We define the elliptic quotient and the rigid quotient as follows:

$$
\mathfrak{R}_{\mathbb{C}}(G)_{\text {ell }}=\mathfrak{R}_{\mathbb{C}}(G) /\left\langle\operatorname{Ind}_{P}^{G}(\sigma)\right)\left|P=M N \in \mathcal{P}, \sigma \in \mathfrak{R}_{\mathbb{C}}(M)\right\rangle ;
$$

$$
\begin{aligned}
& \mathfrak{R}_{\mathbb{C}}(G)_{\text {rig }} \\
& \quad=\mathfrak{R}_{\mathbb{C}}(G) /\left\langle\operatorname{Ind}_{P}^{G}(\sigma)-\operatorname{Ind}_{P}^{G}(\sigma \otimes \chi) \mid P=M N \in \mathcal{P}, \sigma \in \mathfrak{R}_{\mathbb{C}}(M), \chi \in \Psi(M)_{\mathbb{C}}\right\rangle .
\end{aligned}
$$

We have discussed the rigid cocenter $\overline{\mathcal{H}}_{\mathbb{C}}^{\text {rig }}$ in this paper. There is another important subspace of the cocenter, the elliptic cocenter $\overline{\mathcal{H}}_{\mathbb{C}}^{\text {ell }}$, introduced by Bernstein, 
Deligne, and Kazhdan in [5]. By definition,

$$
\overline{\mathcal{H}}_{\mathbb{C}}^{\text {ell }}=\left\{f \in \overline{\mathcal{H}}_{\mathbb{C}} ; \bar{r}_{M}(f)=0 \text { for any proper standard Levi } M\right\},
$$

where $\bar{r}_{M}: \overline{\mathcal{H}}_{\mathbb{C}}^{G} \rightarrow \overline{\mathcal{H}}_{\mathbb{C}}^{M}$ is the map adjunct to the parabolic induction functor $\mathfrak{R}_{\mathbb{C}}(M) \rightarrow \mathfrak{R}_{\mathbb{C}}(G)$.

The trace map $\operatorname{Tr}_{\mathbb{C}}: \overline{\mathcal{H}}_{\mathbb{C}} \rightarrow \mathfrak{R}_{\mathbb{C}}(G)^{*}$ induces

$$
\operatorname{Tr}_{\mathbb{C}}: \overline{\mathcal{H}}_{\mathbb{C}}^{\text {ell }} \rightarrow \mathfrak{R}_{\mathbb{C}}(G)_{\text {ell }}^{*}, \quad \operatorname{Tr}_{\mathbb{C}}: \overline{\mathcal{H}}_{\mathbb{C}}^{\text {rig }} \rightarrow \mathfrak{R}_{\mathbb{C}}(G)_{\text {rig }}^{*}
$$

Here the first map is studied in [5] and the second map is studied in [6].

Similarly, for any $n \in \mathbb{N}$, let $\mathfrak{R}\left(\mathcal{H}_{\mathbb{C}}\left(G, I_{n}\right)\right)$ be the complexified Grothendieck group of finite dimensional representations of $\mathcal{H}_{\mathbb{C}}\left(G, I_{n}\right)$. By $\mathfrak{R}\left(\mathcal{H}_{\mathbb{C}}\left(G, I_{n}\right)\right)_{\text {ell }}$ and $\mathfrak{R}\left(\mathcal{H}_{\mathbb{C}}\left(G, I_{n}\right)\right)_{\text {rig }}$ we denote the elliptic quotient and the rigid quotient of $\mathfrak{R}\left(\mathcal{H}_{\mathbb{C}}(G\right.$, $\left.I_{n}\right)$ ), respectively. Then we have

(b)

$$
\operatorname{Tr}_{\mathbb{C}}: \overline{\mathcal{H}}_{\mathbb{C}}\left(G, I_{n}\right)^{\text {ell }} \rightarrow \mathfrak{R}\left(\mathcal{H}_{\mathbb{C}}\left(G, I_{n}\right)\right)_{\text {ell }}^{*}, \quad \operatorname{Tr}_{\mathbb{C}}: \overline{\mathcal{H}}_{\mathbb{C}}\left(G, I_{n}\right)^{\text {rig }} \rightarrow \mathfrak{R}\left(\mathcal{H}_{\mathbb{C}}\left(G, I_{n}\right)\right)_{\text {rig }}^{*}
$$

If $G$ is semisimple, then all the vector spaces in (b) are finite dimensional and the maps in (b) are bijective. Here the surjectivity follows from the trace Paley-Wiener theorem [5] and [6] and the injectivity follows from the density theorem [16].

5.2. The $P G L_{2}(F)$ case. In this subsection, we assume that $G=P G L_{2}(F)$, where $F$ is a non-archimedean local field with finite residue field $\mathbb{F}_{q}$. We assume furthermore that $q$ is odd.

Up to conjugation, there is

- a unique split maximal torus of $G$, which we denote by $T_{s}$;

- a unique maximal elliptic torus that splits over the unramified extension of $F$, which we denote by $T_{u}$;

- two nonconjugate maximal elliptic tori that split over ramified extensions, which we denote by $T_{r m}$ and $T_{r m}^{\prime}$.

Let $n$ be a positive integer and $r=n-\epsilon$, where $\epsilon$ is sufficiently small. For any subgroup $H$ of $G$, we denote by $[H]$ the set of subgroups of $G$ that are conjugate to $H$. For $H \in\left\{G, T_{s} T_{u}, T_{r m}, T_{r m}^{\prime}\right\}$ and $[\gamma] \in \mathcal{S}_{r}, \sharp_{[H]}$ denotes the cardinality of $\left\{[\gamma] \in \mathcal{S}_{r} \mid\left[C_{G}\left(\gamma_{\leq r}\right)\right]=[H]\right\}$. Then, from the table below, we have

$$
\operatorname{dim} \overline{\mathcal{H}}_{\mathbb{C}}\left(G, I_{n}\right)^{\text {rig }}=3 q^{n}+2 .
$$

\begin{tabular}{|c|c|c|}
\hline$[H]$ & $\sharp[H]$ & $C l_{H}^{u}$ \\
\hline \hline$P G L_{2}$ & 1 & 2 \\
\hline$T_{s}$ & $\frac{q^{n-1}(q-1)}{2}$ & 1 \\
\hline$T_{u}$ & $\frac{q^{n-1}(q+1)}{2}$ & 1 \\
\hline$T_{r m}$ & $q^{n}$ & 1 \\
\hline$T_{r m}^{\prime}$ & $q^{n}$ & 1 \\
\hline
\end{tabular}

Note that every $r^{+}$-equivalence class in $G^{\text {rig }}$ contains some elliptic semisimple element. However, in general, not every $r^{+}$-equivalence class in $G^{\text {rig }}$ consists only of elliptic semisimple elements. One may show that for $G=P G L_{2}$, an $r^{+}$-equivalence class $[\gamma]$ in $G^{\text {rig }}$ consists only of elliptic semisimple elements if and only if $H^{\gamma}$ is a compact subgroup of $G$. We define $\mathcal{S}_{r}^{\text {ell }} \subset \mathcal{S}_{r}$ to be the subset of $r^{+}$-equivalence 
classes in $G^{\text {rig }}$ only consisting of elliptic semisimple elements. Then we have the following identity:

$$
\begin{aligned}
\operatorname{dim} \overline{\mathcal{H}}_{\mathbb{C}}\left(G, I_{n}\right)^{\text {ell }} & =\operatorname{dim} \overline{\mathcal{H}}_{\mathbb{C}}\left(G, I_{n}\right)^{\text {rig }}-\operatorname{dim} \overline{\mathcal{H}}_{\mathbb{C}}\left(T_{s}^{c}, T_{s, n}\right) \\
& =\sharp \mathcal{S}_{r}^{\text {ell }}+1=2 q^{n}+\frac{q^{n-1}(q+1)}{2}+1,
\end{aligned}
$$

where $T_{s}^{c} \subset T_{s}$ is the subgroup consisting of compact elements in $T_{s}, T_{s, n}$ is the $n$th congruent subgroup of $T_{s}$, and the number 1 in the third term comes from the regular unipotent conjugacy class of $G$.

Note that the discrete series gives a natural basis of $\mathcal{R}_{\mathbb{C}}(G)_{\text {ell }}$ and the discrete series of depth at most $r$ gives a natural basis $\mathcal{R}_{\mathbb{C}}\left(\mathcal{H}\left(G, I_{n}\right)\right)_{\text {ell }}$. Moreover, the discrete series consist of supercuspidal representations and four nonsupercuspidal discrete series representations. By direct calculation, one can check the number of supercuspidal representations of depth at most $r$ equals $\sharp \mathcal{S}_{r}^{\text {ell }}-3$, and we have $\operatorname{dim} \overline{\mathcal{H}}_{\mathbb{C}}\left(G, I_{n}\right)^{\text {ell }}=\operatorname{dim} \mathfrak{R}\left(\mathcal{H}_{\mathbb{C}}\left(G, I_{n}\right)\right)_{\text {ell }}$.

5.3. Quaternion algebra. Let $G=P G L_{2}(F)$. Let $D$ be a quaternion algebra over $F$ and $G^{\prime}=D^{\times} / F^{\times}$. It is well known that there is a natural bijection between the set of elliptic semisimple conjugacy classes in $G L_{2}(F)$ and the regular semisimple conjugacy classes in $D^{\times}$. Here $\gamma \leftrightarrow \gamma^{\prime}$ if and only if they have the same characteristic polynomial. Therefore, there is a natural bijection between the set of elliptic semisimple conjugacy classes in $G=P G L_{2}(F)$ and the regular semisimple conjugacy classes in $G^{\prime}$ and this bijection preserves the depth. We have that

$$
\operatorname{dim} \overline{\mathcal{H}}_{\mathbb{C}}\left(G^{\prime}, I_{n}^{G^{\prime}}\right)=\sharp \mathcal{S}_{r}^{G^{\prime}}=\sharp \mathcal{S}_{r}^{G, e l l}+1=\operatorname{dim} \overline{\mathcal{H}}_{\mathbb{C}}\left(G, I_{n}\right)^{\text {ell }} .
$$

Here the number 1 in the third term comes from the $r^{+}$equivalence [1] in $\mathcal{S}_{r}^{G^{\prime}}$.

The local Jacquet-Langlands correspondence [15] gives a bijection between the discrete series of $G$ and the irreducible representations of $G^{\prime}$. The natural duality between the cocenter and representations indicates that there is not only the numerical identity $\operatorname{dim} \overline{\mathcal{H}}_{\mathbb{C}}\left(G^{\prime}, I_{n}^{G^{\prime}}\right)=\operatorname{dim} \overline{\mathcal{H}}_{\mathbb{C}}\left(G, I_{n}\right)^{\text {ell }}$, but there also should be a natural bijection between the cocenter $\overline{\mathcal{H}}_{\mathbb{C}}\left(G^{\prime}, I_{n}^{G^{\prime}}\right)$ and the elliptic cocenter $\overline{\mathcal{H}}_{\mathbb{C}}\left(G, I_{n}\right)^{\text {ell }}$. It would be interesting to study such natural bijections for the (elliptic) cocenters of $P G L_{m}$ and its inner forms for arbitrary $m$.

5.4. The $S L_{2}$ case. For $G=S L_{2}$, here are two nonconjugate elliptic maximal tori that split over unramified extensions, which we denote by $T_{u}$ and $T_{u}^{\prime}$. We have the following table for $S L_{2}$ (with $n=1$ ):

\begin{tabular}{|c|c|c|}
\hline$[H]$ & $\sharp_{[H]}$ & $C l_{H}^{u}$ \\
\hline \hline$S L_{2}$ & 2 & 5 \\
\hline$T_{s}$ & $\frac{q-3}{2}$ & 1 \\
\hline$T_{u}$ & $\frac{q-1}{2}$ & 1 \\
\hline$T_{u}^{\prime}$ & $\frac{q-1}{2}$ & 1 \\
\hline$T_{r m}$ & $q-1$ & 1 \\
\hline$T_{r m}^{\prime}$ & $q-1$ & 1 \\
\hline
\end{tabular}

We have

$$
\operatorname{dim} \overline{\mathcal{H}}_{\mathbb{C}}\left(G, I_{n}\right)^{\text {rig }}=3 q+6+\frac{q-1}{2}, \quad \operatorname{dim} \overline{\mathcal{H}}_{\mathbb{C}}\left(G, I_{n}\right)^{\text {ell }}=3 q+5 .
$$


We have seen that for $G=P G L_{2}, \operatorname{dim} \overline{\mathcal{H}}_{\mathbb{C}}\left(G, I_{1}\right)^{\text {rig }}=3 q+2$ and $\operatorname{dim} \overline{\mathcal{H}}_{\mathbb{C}}\left(G, I_{n}\right)^{\text {ell }}=$ $2 q+\frac{q+3}{2}$. Thus the elliptic/rigid cocenters for $P G L_{2}$ and $S L_{2}$ are different.

\section{ACKNOWLEDGMENTS}

We would like to thank Anne-Marie Aubert and Jessica Fintzen for their valuable comments, and Jeff Adler for helpful correspondence. We would like to thank Roger Howe for his question which led the authors to add $\S 5$ of this paper. We would also like to thank the referee for the careful reading and valuable comments.

This work was done while both authors were members at the Institute for Advanced Study during 2016-1017. We would like to thank them for their hospitality and support.

\section{REFERENCES}

[1] Jeffrey D. Adler, Refined anisotropic K-types and supercuspidal representations, Pacific J. Math. 185 (1998), no. 1, 1-32, DOI 10.2140/pjm.1998.185.1. MR.1653184

[2] Jeffrey D. Adler and Stephen DeBacker, Some applications of Bruhat-Tits theory to harmonic analysis on the Lie algebra of a reductive p-adic group, Michigan Math. J. 50 (2002), no. 2, 263-286, DOI 10.1307/mmj/1028575734. MR.1914065

[3] Jeffrey D. Adler and Loren Spice, Good product expansions for tame elements of p-adic groups, Int. Math. Res. Pap. IMRP 1 (2008), Art. ID rp. 003, 95. MR2431235

[4] Dan Barbasch and Allen Moy, A new proof of the Howe conjecture, J. Amer. Math. Soc. 13 (2000), no. 3, 639-650, DOI 10.1090/S0894-0347-00-00336-2. MR1758757

[5] J. Bernstein, P. Deligne, and D. Kazhdan, Trace Paley-Wiener theorem for reductive p-adic groups, J. Analyse Math. 47 (1986), 180-192, DOI 10.1007/BF02792538. MR874050

[6] D. Ciubotaru and X. He, Cocenters of p-adic groups, III: Elliptic cocenter and rigid cocenter, arXiv:1703.00378.

[7] Laurent Clozel, Orbital integrals on p-adic groups: a proof of the Howe conjecture, Ann. of Math. (2) 129 (1989), no. 2, 237-251, DOI 10.2307/1971447. MR986793

[8] Stephen DeBacker, Parametrizing nilpotent orbits via Bruhat-Tits theory, Ann. of Math. (2) 156 (2002), no. 1, 295-332, DOI 10.2307/3597191. MR.1935848

[9] Stephen DeBacker, Homogeneity results for invariant distributions of a reductive p-adic group (English, with English and French summaries), Ann. Sci. École Norm. Sup. (4) 35 (2002), no. 3, 391-422, DOI 10.1016/S0012-9593(02)01094-7. MR1914003

[10] J. Fintzen, Tame tori in p-adic groups and good semisimple elements, arXiv:1801.04955.

[11] Thomas C. Hales, A simple definition of transfer factors for unramified groups, Representation theory of groups and algebras, Contemp. Math., vol. 145, Amer. Math. Soc., Providence, RI, 1993, pp. 109-134, DOI 10.1090/conm/145/1216184. MR.1216184

[12] Xuhua He, Cocenters of p-adic groups, I: Newton decomposition, Forum Math. Pi 6 (2018), e2, 27, DOI 10.1017/fmp.2018.1. MR3780377

[13] Xuhua He, Cocenter of p-adic groups, II: induction map, Adv. Math. 345 (2019), 972-997, DOI 10.1016/j.aim.2019.01.039. MR.3903646

[14] Roger Howe, Two conjectures about reductive p-adic groups, Harmonic analysis on homogeneous spaces (Proc. Sympos. Pure Math., Vol. XXVI, Williams Coll., Williamstown, Mass., 1972), Amer. Math. Soc., Providence, R.I., 1973, pp. 377-380. MR0338278

[15] H. Jacquet and R. P. Langlands, Automorphic forms on GL(2), Lecture Notes in Mathematics, Vol. 114, Springer-Verlag, Berlin-New York, 1970. MR0401654

[16] David Kazhdan, Cuspidal geometry of p-adic groups, J. Analyse Math. 47 (1986), 1-36, DOI 10.1007/BF02792530. MR874042

[17] Ju-Lee Kim, Supercuspidal representations: an exhaustion theorem, J. Amer. Math. Soc. 20 (2007), no. 2, 273-320, DOI 10.1090/S0894-0347-06-00544-3. MR2276772

[18] Ju-Lee Kim and Fiona Murnaghan, Character expansions and unrefined minimal K-types, Amer. J. Math. 125 (2003), no. 6, 1199-1234. MR2018660

[19] Ju-Lee Kim and Fiona Murnaghan, K-types and $\Gamma$-asymptotic expansions, J. Reine Angew. Math. 592 (2006), 189-236, DOI 10.1515/CRELLE.2006.027. MR2222734 
[20] George J. McNinch, Nilpotent orbits over ground fields of good characteristic, Math. Ann. 329 (2004), no. 1, 49-85, DOI 10.1007/s00208-004-0510-9. MR2052869

[21] Allen Moy and Gopal Prasad, Unrefined minimal K-types for p-adic groups, Invent. Math. 116 (1994), no. 1-3, 393-408, DOI 10.1007/BF01231566. MR1253198

[22] Allen Moy and Gopal Prasad, Jacquet functors and unrefined minimal K-types, Comment. Math. Helv. 71 (1996), no. 1, 98-121, DOI 10.1007/BF02566411. MR1371680

[23] Gopal Prasad, Galois-fixed points in the Bruhat-Tits building of a reductive group (English, with English and French summaries), Bull. Soc. Math. France 129 (2001), no. 2, 169-174, DOI 10.24033/bsmf.2391. MR.1871292

[24] R. Ranga Rao, Orbital integrals in reductive groups, Ann. of Math. (2) 96 (1972), 505-510, DOI 10.2307/1970822. MR0320232

[25] Guy Rousseau, Immeubles des groupes réducitifs sur les corps locaux (French), U.E.R. Mathématique, Université Paris XI, Orsay, 1977. Thèse de doctorat; Publications Mathématiques d'Orsay, No. 221-77.68. MR0491992

[26] Loren Spice, Topological Jordan decompositions, J. Algebra 319 (2008), no. 8, 3141-3163, DOI 10.1016/j.jalgebra.2007.11.004. MR2408311

Department of Mathematics, University of Maryland, College Park, Maryland 20742 Current address: The Institute of Mathematical Sciences and Department of Mathematics, The Chinese University of Hong Kong, Shatin, N.T., Hong Kong.

Email address: xuhuahe@math.cuhk.edu.hk

Department of Mathematics, Massachusetts Institute of Technology, 77 Massachusetts Avenue, Cambridge Massachusetts 02139

Email address: juleekim@mit.edu 